\title{
Equivariant Hopf Galois extensions and Hopf cyclic cohomology
}

\author{
Mohammad Hassanzadeh and Bahram Rangipour
}

\begin{abstract}
We define the notion of equivariant $\times$-Hopf Galois extension and apply it as a functor between the categories of stable anti-Yetter-Drinfeld (SAYD) modules of the $\times$-Hopf algebras involved in the extension. This generalizes the result of Jara-Ştefan and Böhm-Ştefan on associating a SAYD modules to any ordinary Hopf Galois extension.
\end{abstract}

Mathematics Subject Classification (2010). 19D55, 16T05, 16S40.

Keywords. Galois extension, $\times$-Hopf algebras, SAYD modules, Hopf cyclic cohomology.

\section{Introduction}

Hopf cyclic cohomology, which is now a well-known cohomology theory in noncommutative geometry, was invented by A. Connes and H. Moscovici in [CM98] as a computational tool for calculating the local index formula of spectral triples associated to hypoecliptic operators on manifolds. Since then, the theory has been evolving to cover many other cases including algebras and coalgebras endowed with (co)symmetry from Hopf algebras, bialgebras, and Hopf algebras with several objects such as para-Hopf algebras and $\times$-Hopf algebras [CM00], [CM01], [BS], [Cra], [Gor], [HKRS1], [HKRS2], [JS], [Kay05], [Kay06], [KP], [KR02], [KR05], [KR04], [KR03], [Ra]. The main application of Hopf cyclic cohomology is to produce special cyclic cocycles on (co)algebras endowed with a (co)symmetry by a Hopf algebra. Generally speaking, Hopf cyclic cohomology of a Hopf algebra is more computable than cyclic cohomology of (co)algebras upon which the Hopf algebra (co)acts. This accessibility is the main motivation and application of Hopf cyclic cohomology. The Hopf cyclic cohomology of Hopf (co)module (co)algebras with coefficients in SAYD modules was first defined in [HKRS2], [HKRS1], where it was shown that the modular pair in involution defined by Connes and Moscovici in [CM00] is in fact, a onedimensional SAYD module. Later on, Jara and Ştefan in [JS] associated a canonical SAYD module to any Hopf-Galois extension such that the relative cyclic complex of the extension is isomorphic to the cyclic complex defined on the Hopf algebra with coefficients in the associated SAYD module. Soon after, Khalkhali and the second author showed in [KR05] that the cyclic module obtained by Jara-Ştefan is of the 
form of a cyclic module, defined in [KR02], which is dual (in the sense of Connes' cyclic category) to the Connes-Moscovici cocyclic module.

The first step for defining Hopf cyclic cohomology of Hopf algebras with several objects was taken in [CM01], where it was shown, among other things, that the symmetry on the crossed product algebra of the diffeomorphism group and the algebra of smooth functions on the frame bundle of a not necessarily flat manifold is defined by a Hopf algebroid generalizing the Connes-Moscovici Hopf algebra in the case the manifold in question is flat. The cohomology defined in [CM01] was abstractly generalized for a class of Hopf algebroids called para-Hopf algebras in [KR04]. The Hopf cyclic cohomology of a coring with symmetry from a para-Hopf algebra was defined in [Ra]. Finally Hopf cyclic cohomology of $\times$-Hopf algebras with coefficients in the SAYD modules was defined by Böhm and Ştefan in [BS], where it was also shown that the result of Jara-Ştefan is valid for $\times$-Hopf Galois extensions.

Let us recall very briefly here the result of Jara-Ştefan from [JS]. Let $\mathbf{\nabla}: A \rightarrow$ $A \otimes H$ define a comodule algebra and let $B$ be the subalgebra of coinvariants for this coaction. Then the extension $B \subseteq A$ is called Galois if the canonical map can: $A \otimes_{B} A \rightarrow A \otimes H$ is bijective. One uses the map can iteratively to transfer the cyclic structure on the relative cyclic complex of $B \subset A$ to get a cyclic module on the other side depending only on the Hopf algebra $H$ and the SAYD module $A_{B}:=A /[A, B]$.

In this paper we start from the fact that relative cyclic homology of an extension $B \subseteq A$ is in fact Hopf cyclic homology of the ring $A$ over $B$ with coefficients in $B$ as a SAYD module over the $\times_{B}$-Hopf algebra $B^{e}:=B \otimes B^{\text {op }}$. It is also easy to observe that $A_{B}=B \otimes_{B^{e}} A$. On the other hand, if $M$ is a SAYD module over $B^{e}$, in the sense of [BS], then the cyclic homology of the $B$-ring $A$ with coefficients in $M$ under symmetry of $B^{e}$ is well defined. So in case the extension is $H$-Galois iterative application of the map can takes us to a cyclic complex which is again the dual of the Connes-Moscovici cocyclic module associated to the Hopf algebra $H$ with coefficients in $M \otimes_{B} e A$. The mentioned transfer of cyclic structures works due to the fact that the map can happens to be $B^{e}$-equivariant. This means that if one assumes the existence of a $\times$ Hopf algebra $\mathcal{K}$ acting on $A$ such that the map can is equivariant, then to any SAYD module $M$ over $\mathcal{K}$ one associates a SAYD module $\widetilde{M}:=M \otimes \mathcal{K} A$ over $H$. One then shows that the Hopf cyclic homology of the ring $A$ under symmetry of $\mathcal{K}$ with coefficients in $M$ is isomorphic to the dual cyclic module of the Hopf cocyclic module of $H$ with coefficients in $\tilde{M}$. The next step is to upgrade everything to the level of a $\times$-Hopf Galois extension, which is stated as Theorem 3.5.

The plan of the paper is as follows. In Section 1 we recall the basics of co(cyclic) modules and duality in the cyclic category. In Section 2 we review the concepts of left and right $\times$-Hopf algebras and basics of Hopf cyclic (co)homologies together with some examples. In Section 3 we define the equivariant $\times$-Hopf Galois extensions and prove the main result of the paper which is summarized in Theorem 3.5 and finally we bring some non-trivial examples of this result. 
Throughout the paper we assume that all objects are $\mathbb{C}$-vector spaces although everything works for $k$-modules, where $k$ is a commutative ring. We use the Sweedler summations: for comultiplication of coalgebras or corings, i.e., $\Delta(c)=c^{(1)} \otimes c^{(2)}$ for coactions, i.e, $\mathbf{\nabla}(m)=m_{\langle 0\rangle} \otimes m_{\langle 1\rangle}$; for the translation map, i.e, $\operatorname{can}^{-1}(b)=b_{\langle-\rangle} \otimes$ $b_{\langle+\rangle}$; and finally for the "antipode" of $\times$-Hopf algebras, i.e, $v^{-1}(1 \otimes b)=b^{-} \otimes b^{+}$.

Acknowledgement. The authors would like to thank the referee for carefully reading the manuscript and also for valuable comments.

\section{Basics of (co)cyclic modules}

In this section we recall the definitions of cyclic and cocyclic modules from [C-Book] (see also [Lo]). Recall that a cosimplicial module is given by a datum $\left(C_{n}, \delta_{i}, \sigma_{i}\right)$ where $C_{n}, n \geq 0$, is a $\mathbb{C}$-module and the maps $\delta_{i}: C^{n} \rightarrow C^{n+1}, \sigma_{i}: C^{n} \rightarrow C^{n-1}$ are called cofaces and codegeneracies, respectively. These are $\mathbb{C}$-module maps satisfying the following cosimplicial relations:

$$
\begin{aligned}
& \delta_{j} \delta_{i}=\delta_{i} \delta_{j-1} \quad \text { if } i<j, \\
& \sigma_{j} \sigma_{i}=\sigma_{i} \sigma_{j+1} \text { if } i \leq j, \\
& \sigma_{j} \delta_{i}= \begin{cases}\delta_{i} \sigma_{j-1} & \text { if } i<j, \\
\mathrm{Id} & \text { if } i=j \text { or } i=j+1, \\
\delta_{i-1} \sigma_{j} & \text { if } i>j+1 .\end{cases}
\end{aligned}
$$

A cocyclic module is a cosimplicial module equipped with extra morphisms, $\tau: C^{n} \rightarrow$ $C^{n}$, called cocyclic maps, such that the following extra relations hold.

$$
\begin{array}{rlrl}
\tau \delta_{i}=\delta_{i-1} \tau, & & 1 \leq i \leq n+1, \\
\tau \delta_{0}=\delta_{n+1}, & & 1 \leq i \leq n, \\
\tau \sigma_{i}=\sigma_{i-1} \tau, & 1 \leq i \leq n, \\
\tau \sigma_{0}=\sigma_{n} \tau^{2}, & \tau^{n+1}=\text { Id. }
\end{array}
$$

In a dual manner, one can define a cyclic module as a simplicial module with extra cyclic maps. More precisely: A cyclic module is given by a datum $C=$ $\left(C_{n}, \delta_{i}, \sigma_{i}, \tau_{n}\right)$, where $C_{n}, n \geq 0$, is a $\mathbb{C}$-module and $\delta_{i}: C_{n} \rightarrow C_{n-1}, \sigma_{i}: C_{n} \rightarrow$ $C_{n+1}, 0 \leq i \leq n$, and $\tau: C_{n} \rightarrow C_{n}$, called faces, degeneracies and cyclic maps, respectively, are $\mathbb{C}$-module maps satisfying the following relations:

$$
\begin{aligned}
& \delta_{i} \delta_{j}=\delta_{j-1} \delta_{i} \quad \text { if } i<j, \\
& \sigma_{i} \sigma_{j}=\sigma_{j+1} \sigma_{i} \quad \text { if } i \leq j, \\
& \delta_{i} \sigma_{j}= \begin{cases}\sigma_{j-1} \delta_{i} & \text { if } i<j, \\
\mathrm{Id} & \text { if } i=j \text { or } i=j+1, \\
\sigma_{j} \delta_{i-1} & \text { if } i>j+1,\end{cases}
\end{aligned}
$$


and

$$
\begin{aligned}
\delta_{i} \tau & =\tau \delta_{i-1}, & & 1 \leq i \leq n, \\
\delta_{0} \tau & =\delta_{n}, & & 1 \leq i \leq n, \\
\sigma_{i} \tau & =\tau \sigma_{i-1}, & & 1 \leq i \leq n, \\
\sigma_{0} \tau & =\tau^{2} \sigma_{n}, & & \\
\tau^{n+1} & =\mathrm{Id} . & &
\end{aligned}
$$

Remark 1.1 (Duality). Let $C=\left(C^{n}, d_{i}, s_{i}, t\right)$ be a cocyclic module. We denote its cyclic dual by $\widetilde{C}$. It is shown in [C-Book] that, with the following operators, $\widetilde{C}_{n}:=C^{n}$ is a cyclic module.

$$
\begin{array}{rlrl}
\delta_{i} & :=s_{i}: \widetilde{C}_{n} \rightarrow \widetilde{C}_{n-1}, & 0 \leq i \leq n-1, \\
\delta_{n} & :=\delta_{0} \tau_{n}, \\
\sigma_{i} & :=d_{i+1}: \widetilde{C}_{n} \rightarrow \widetilde{C}_{n+1}, \quad 0 \leq i \leq n-1, \\
\tau & :=t^{-1} .
\end{array}
$$

Conversely, one obtains a cocyclic module from a cyclic module as follows. Let $C=\left(C_{n}, \delta_{i}, \sigma_{i}, \tau\right)$ be a cyclic module. Its cyclic dual is denoted by $\check{C}$ and defined by $\breve{C}^{n}=C_{n}$ with the following cofaces, codegeneracies and cyclic operator.

$$
\begin{aligned}
d_{0} & :=\tau_{n} \sigma_{n-1}, & & 1 \leq i \leq n, \\
d_{i} & :=\sigma_{i-1}: \check{C}^{n} \rightarrow \check{C}^{n+1}, & & 1 \leq i \leq n, \\
s_{i} & :=\delta_{i}: \check{C}^{n} \rightarrow \check{C}^{n-1}, & & 0 \leq i \leq n-1, \\
t & :=\tau^{-1} . & &
\end{aligned}
$$

\section{Hopf cyclic homology of $\mathrm{x}_{R}$-Hopf algebras}

We refer the reader to $[\mathrm{B}],[\mathrm{BW}],[\mathrm{Sch}],[\mathrm{Sch} 98]$ for details about bialgebroids and $\times$-Hopf algebras, however in Section 2.1 we recall the basics of $\times_{R}$-Hopf algebras. We review the stable anti-Yetter-Drinfeld modules for $\times_{R}$-Hopf algebras in Section 2.2 from [BS]. Finally in Section 2.3 we bring the cyclic modules of module algebras and module corings over $\times_{R}$-Hopf algebras with coefficients in stable antiYetter-Drinfeld modules.

2.1. Preliminaries of $\mathbf{x}_{\boldsymbol{R}}$-Hopf algebras. Let $R$ and $\mathcal{K}$ be algebras over $\mathbb{C}$. In addition let the source and the target maps be the $\mathbb{C}$-algebra homomorphisms $\mathfrak{s}: R \rightarrow \mathcal{K}$ and $\mathrm{t}: R^{\mathrm{op}} \rightarrow \mathcal{K}$ such that their ranges commute with one another. We equip $\mathcal{K}$ with an $R$-bimodule structure by $r_{1} k r_{2}=\mathfrak{s}\left(r_{1}\right) \mathrm{t}\left(r_{2}\right) k$ for $r_{1}, r_{2} \in R$ and $k \in \mathcal{K}$. Similarly $\mathcal{K} \otimes_{R} \mathcal{K}$ is endowed with the natural $R$-bimodule structure, 
i.e, $r_{1}\left(k_{1} \otimes_{R} k_{2}\right) r_{2}:=\mathfrak{s}\left(r_{1}\right) k_{1} \otimes_{R} \mathrm{t}\left(r_{2}\right) k_{2}$. We assume that there are $R$-bimodule maps, called the coproduct $\Delta: \mathcal{K} \rightarrow \mathcal{K} \otimes_{R} \mathcal{K}$ and the couint $\varepsilon: \mathcal{K} \rightarrow R$, via which $\mathcal{K}$ is an $R$-coring. The datum $(\mathcal{K}, \mathfrak{s}, \mathrm{t}, \Delta, \varepsilon)$ is called a left $R$-bialgebroid if for $k_{1}, k_{2} \in \mathcal{K}$ and $r \in R$ the following identities hold:

i) $k^{(1)} \mathrm{t}(r) \otimes_{R} k^{(2)}=k^{(1)} \otimes_{R} k^{(2)} \mathfrak{s}(r)$,

ii) $\Delta\left(1_{\mathcal{K}}\right)=1_{\mathcal{K}} \otimes_{R} 1_{\mathcal{K}}$ and $\Delta\left(k_{1} k_{2}\right)=k_{1}^{(1)} k_{2}^{(1)} \otimes_{R} k_{1}^{(2)} k_{2}^{(2)}$,

iii) $\varepsilon\left(1_{\mathcal{K}}\right)=1_{R}$ and $\varepsilon\left(k_{1} k_{2}\right)=\varepsilon\left(k_{1} \mathfrak{s}\left(\varepsilon\left(k_{2}\right)\right)\right)$.

For a left bialgebroid $\mathcal{K}$ over an algebra $R$, the following identities hold for $r, r_{1}, r_{2}, r_{3}, r_{4} \in R$ and $k, k_{1}, k_{2} \in \mathcal{K}$ [BW], 4.31:

a) $\varepsilon(\mathfrak{s}(r))=\varepsilon(\mathrm{t}(r))=r$,

b) $s\left(\varepsilon\left(k^{(1)}\right)\right) k^{(2)}=\mathrm{t}\left(\varepsilon\left(k^{(2)}\right)\right) k^{(1)}=k$,

c) $\varepsilon\left(k_{1} k_{2}\right)=\varepsilon\left(k_{1} \mathrm{t}\left(\varepsilon\left(k_{2}\right)\right)\right)$,

d) $\Delta(\mathfrak{s}(r))=\mathfrak{s}(r) \otimes_{R} 1_{\mathcal{K}}$ and $\Delta(\mathrm{t}(r))=1_{\mathcal{K}} \otimes_{R} \mathrm{t}(r)$,

e) $\Delta\left(\mathfrak{s}\left(r_{1}\right) \mathrm{t}\left(r_{2}\right) k \mathfrak{s}\left(r_{3}\right) t\left(r_{4}\right)\right)=\mathfrak{s}\left(r_{1}\right) k^{(1)} \mathfrak{s}\left(r_{3}\right) \otimes_{R} \mathrm{t}\left(r_{2}\right) k^{(2)} \mathrm{t}\left(r_{4}\right)$.

Similarly a right $R$-bialgebroid $\mathscr{B}$ is an $R$-coring $(\mathscr{B}, \mathfrak{s}, \mathrm{t}, \Delta, \varepsilon)$, where $s, \mathrm{t}: R \rightarrow$ $\mathscr{B}$ are algebra and anti-algebra homomorphisms with commuting ranges, and $\mathscr{B}$ is an $R$-bimodule with the right multiplications by $\mathfrak{s}$ and $\mathrm{t}$ such that the following conditions are satisfied for $b, b_{1}, b_{2} \in \mathscr{B}$ and $r \in R$ :

i) $b^{(1)} \otimes_{R} \mathrm{t}(r) b^{(2)}=\mathfrak{s}(r) b^{(1)} \otimes_{R} b^{(2)}$,

ii) $\Delta\left(1_{B}\right)=1_{B} \otimes_{R} 1_{B}$ and $\Delta\left(b_{1} b_{2}\right)=b_{1}^{(1)} b_{2}^{(1)} \otimes_{R} b_{1}^{(2)} b_{2}^{(2)}$,

iii) $\varepsilon\left(1_{B}\right)=1_{R}$ and $\varepsilon\left(b_{1} b_{2}\right)=\varepsilon\left(\mathfrak{s}\left(\varepsilon\left(b_{1}\right)\right) b_{2}\right)$.

For a right bialgebroid $\mathscr{B}$ over an algebra $R$, the following identities hold for $r, r_{1}, r_{2}, r_{3}, r_{4} \in R$ and $b, b_{1}, b_{2} \in B$ :

a) $\varepsilon(\mathfrak{s}(r))=\varepsilon(\mathrm{t}(r))=r$,

b) $b=b^{(1)} \mathfrak{s}\left(\varepsilon\left(b^{(2)}\right)\right)=b^{(2)} \mathrm{t}\left(\varepsilon\left(b^{(1)}\right)\right)$,

c) $\varepsilon\left(b_{1} b_{2}\right)=\varepsilon\left(\mathrm{t}\left(\varepsilon\left(b_{1}\right)\right) b_{2}\right)$,

d) $\Delta(\mathfrak{s}(r))=1_{B} \otimes_{R} \mathfrak{s}(r)$ and $\Delta(\mathrm{t}(r))=\mathrm{t}(r) \otimes_{R} 1_{B}$,

e) $\Delta\left(\mathfrak{s}\left(r_{1}\right) \mathrm{t}\left(r_{2}\right) b \mathfrak{s}\left(r_{3}\right) \mathrm{t}\left(r_{4}\right)\right)=\mathrm{t}\left(r_{2}\right) b^{(1)} \mathrm{t}\left(r_{4}\right) \otimes_{R} \mathfrak{s}\left(r_{1}\right) b^{(2)} \mathfrak{s}\left(r_{3}\right)$.

A left $R$-bialgebroid $(\mathcal{K}, \mathfrak{s}, \mathrm{t}, \Delta, \varepsilon)$ is said to be a left $\times_{R}$-Hopf algebra provided that the map

$$
v: \mathcal{K} \otimes_{R^{\text {op }}} \mathcal{K} \rightarrow \mathcal{K} \otimes_{R} \mathcal{K}, \quad k \otimes_{R^{\text {op }}} k^{\prime} \mapsto k^{(1)} \otimes_{R} k^{(2)} k^{\prime},
$$

is bijective. Here on the left-hand side of (2.1) the $R^{\text {op }}$-module structures are given by right and left multiplication by $\mathrm{t}(r)$ for $r \in R$, however on the right-hand side the $R$-module structures are given by the original actions of $R$ on $\mathcal{K}$. One notes that $v$ and $v^{-1}$ are right $\mathcal{K}$-linear. The role of antipode in $\times_{R}$-Hopf algebras is played by 
the map

$$
\mathcal{K} \rightarrow \mathcal{K} \otimes_{R^{\text {op }}} \mathcal{K}, \quad k \mapsto v^{-1}\left(k \otimes_{R} 1 \mathcal{K}\right) .
$$

For convenience we use $k^{-} \otimes_{R^{\text {op }}} k^{+}$for $v^{-1}\left(k \otimes_{R} 1_{\mathcal{K}}\right)$.

Example 2.1. Let $R$ be an algebra over $\mathbb{C}$. The simplest left $\times_{R}$-Hopf algebra is $\mathcal{K}=R \otimes R^{\mathrm{op}}$ with the source and target maps defined by

$$
\mathfrak{s}: R \rightarrow \mathcal{K}, r \mapsto r \otimes 1, \quad \mathrm{t}: R^{\mathrm{op}} \rightarrow \mathcal{K}, r \mapsto 1 \otimes r
$$

comultiplication defined by

$$
\Delta: \mathcal{K} \rightarrow \mathcal{K} \otimes_{R} \mathcal{K}, \quad r_{1} \otimes r_{2} \mapsto\left(r_{1} \otimes 1\right) \otimes_{R}\left(1 \otimes r_{2}\right),
$$

counit given by

$$
\varepsilon: \mathcal{K} \rightarrow R, \quad \varepsilon\left(r_{1} \otimes r_{2}\right)=r_{1} r_{2}
$$

and

$$
\begin{aligned}
v\left(\left(r_{1} \otimes r_{2}\right) \otimes\left(r_{3} \otimes r_{4}\right)\right) & =r_{1} \otimes 1 \otimes r_{3} \otimes r_{4} r_{2}, \\
v^{-1}\left(\left(r_{1} \otimes r_{2}\right) \otimes\left(r_{3} \otimes r_{4}\right)\right) & =r_{1} \otimes 1 \otimes r_{2} r_{3} \otimes r_{4},
\end{aligned}
$$

where $r, r_{1}, r_{2}, r_{3}, r_{4} \in R$.

The following properties are a symmetrical version of [Sch], Proposition 3.7. We refer the reader to [BS], Lemma 2.14, for more properties of a right $\times$-Hopf algebras.

Proposition 2.2. Let $R$ be an algebra over $\mathbb{C}$ and $\mathcal{K}$ a left $\times_{R}$-Hopf algebra. The following identities hold for $k, k^{\prime} \in \mathcal{K}$ and $r \in R$ :

i) $k^{-(1)} \otimes_{R} k^{-(2)} k^{+}=k \otimes_{R} 1 \mathcal{K}$.

ii) $k^{(1)^{-}} \otimes_{R^{\mathrm{op}}} k^{(1)^{+}} k^{(2)}=k \otimes_{R^{\mathrm{op}}} 1 \mathcal{K}$.

iii) $\left(k k^{\prime}\right)^{-} \otimes_{R^{\text {op }}}\left(k k^{\prime}\right)^{+}=k^{-} k^{\prime-} \otimes_{R_{\text {op }}} k^{\prime+} k^{+}$.

iv) $1_{\mathcal{K}}^{-} \otimes_{R^{\text {op }}} 1_{\mathcal{K}}^{+}=1_{\mathcal{K}} \otimes_{R^{\text {op }}} 1_{\mathcal{K}}$.

v) $k^{-(1)} \otimes_{R} k^{-(2)} \otimes_{R^{\mathrm{op}}} k^{+}=k^{(1)} \otimes_{R} k^{(2)^{-}} \otimes_{R^{\mathrm{op}}} k^{(2)^{+}}$.

vi) $k^{-} \otimes_{R^{\text {op }}} k^{+(1)} \otimes_{R} k^{+(2)}=k^{--} \otimes_{R^{\text {op }}} k^{+} \otimes_{R} k^{-+}$.

vii) $k=k^{-} \mathrm{t}\left(\varepsilon\left(k^{+}\right)\right)$.

viii) $\mathfrak{s}(\varepsilon(k))=k^{-} k^{+}$.

ix) $k^{-} \otimes_{R^{\text {op }}} k^{+} \mathrm{t}(r)=\mathrm{t}(r) k^{-} \otimes_{R^{\text {op }}} k^{+}$.

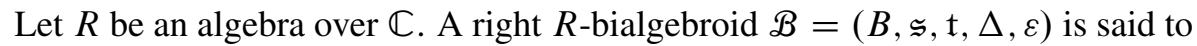
be a right $\times_{R}$-Hopf algebra provided that the map

$$
\nu: \mathscr{B} \otimes_{R^{\text {op }}} \mathcal{B} \rightarrow \mathscr{B} \otimes_{R} \mathcal{B}, \quad b \otimes_{R^{\text {op }}} b^{\prime} \mapsto b b^{(1)} \otimes_{R} b^{(2)},
$$


is bijective. In the domain of the map (2.2), $R^{\text {op }}$-right and left module structures of $\mathscr{B}$ are given by right and left multiplication by $\mathrm{t}(r)$ respectively, for $r \in R$. In the codomain of the map (2.2), $R$-module structures are given by right multiplication by $\mathfrak{s}(r)$ and $\mathrm{t}(r)$.

One notes that $v$ and $v^{-1}$ are left $\mathscr{B}$-linear maps. We denote the image of $v^{-1}$ by the index notation

$$
v^{-1}\left(1 \otimes_{R^{\text {op }}} b\right)=b^{-} \otimes_{R} b^{+} .
$$

Example 2.3. Let $R$ be an algebra over $\mathbb{C}$. The $\mathscr{B}=R \otimes R^{\text {op }}$ is a right $\times_{R}$-Hopf algebra where the source and target maps are defined by

$$
\mathfrak{s}: R \rightarrow \mathscr{B}, r \mapsto r \otimes 1, \quad \mathrm{t}: R^{\mathrm{op}} \rightarrow \mathscr{B}, r \mapsto 1 \otimes r,
$$

comultiplication by

$$
\Delta: \mathscr{B} \rightarrow \mathscr{B} \otimes_{B} \mathcal{B}, \quad r_{1} \otimes r_{2} \mapsto\left(1 \otimes r_{2}\right) \otimes_{B}\left(r_{1} \otimes 1\right),
$$

counit by

$$
\varepsilon: \mathscr{B}^{e} \rightarrow B, \quad \varepsilon\left(r_{1} \otimes r_{2}\right)=r_{2} r_{1},
$$

and

$$
\begin{aligned}
v\left(\left(r_{1} \otimes r_{2}\right) \otimes\left(r_{3} \otimes r_{4}\right)\right) & =r_{1} \otimes r_{4} r_{2} \otimes r_{3} \otimes 1, \\
v^{-1}\left(\left(r_{1} \otimes r_{2}\right) \otimes\left(r_{3} \otimes r_{4}\right)\right) & =r_{1} r_{4} \otimes r_{2} \otimes r_{3} \otimes 1 .
\end{aligned}
$$

2.2. Stable anti-Yetter-Drinfeld-modules. In this subsection for the reader's convenience we briefly recall the definitions of module, comodule and stable anti-YetterDrinfeld-module for $\times_{R}$-Hopf algebras. Also we present an example of a stable antiYetter-Drinfeld (SAYD) module for the $\times_{R}$-Hopf algebra $R \otimes R^{\text {op }}$ which plays an important role in the sequel section.

A right module over a $\times_{R}$-Hopf algebra $\mathcal{K}$ is a right $\mathcal{K}$-module $M$ which is naturally an $R$-bimodule by $r m=m \triangleleft \mathrm{t}(r)$ and $m r=m \triangleleft \mathfrak{s}(r)$. Similarly, by a left module over a right $\times_{R}$-Hopf algebra $\mathcal{B}$ we mean a left $\mathcal{B}$-module $M$, which is naturally an $R$-bimodule by $r m=\mathfrak{s}(r) \triangleright m$ and $m r=\mathrm{t}(r) \triangleright m$.

A left (right) comodule over an $R$-coring $\ell$ is defined by a left(right) $R$-module $M$ together with a left(right) $R$-module coaction map,

$$
M \rightarrow \mathcal{\ell} \otimes_{R} M, \quad m \mapsto m_{\langle-1\rangle} \otimes_{R} m_{\langle 0\rangle},
$$

$\left(M \rightarrow M \otimes_{R} \mathcal{C}, m \mapsto m_{\langle 0\rangle} \otimes_{R} m_{\langle 1\rangle}\right)$ satisfying the coassociativity and counitality axioms.

By a comodule over a $\times$-Hopf algebra we mean a comodule over the underlying coring. One notes that a left comodule $M$ of a left bialgebroid $\mathcal{K}$ can be equipped with an $R$-bimodule structure by introducing a right $R$-action

$$
m r:=\varepsilon\left(m_{\langle-1\rangle} \mathfrak{s}(r)\right) \triangleright m_{\langle 0\rangle}
$$


for $r \in R$ and $m \in M$. It is checked in [BS] that the left $\mathcal{K}$-coaction on the left comodule $M$ is an $R$-bimodule map. That is, for $r, r^{\prime} \in R$ and $m \in M$,

$$
\left(r m r^{\prime}\right)_{\langle-1\rangle} \otimes_{R}\left(r m r^{\prime}\right)_{\langle 0\rangle}=\mathfrak{s}(r) m_{\langle-1\rangle} \mathfrak{s}\left(r^{\prime}\right) \otimes_{R} m_{\langle 0\rangle} .
$$

Furthermore, for any $m \in M$ and $r \in R$,

$$
m_{\langle-1\rangle} \otimes_{R} m_{\langle 0\rangle} r=m_{\langle-1\rangle} \mathrm{t}(r) \otimes_{R} m_{\langle 0\rangle} .
$$

Here we recall the definition of stable anti-Yetter-Drinfeld modules over $\times$-Hopf algebras from [BS]. Let $\mathcal{K}$ be a left $\times_{R}$-Hopf algebra and $M$ be a right $\mathcal{K}$-module and a left $\mathcal{K}$-comodule. We say $M$ is a right-left anti-Yetter-Drinfeld module provided that the following conditions hold:

i) The $R$-bimodule structures on $M$, underlying its module and comodule structures, coincide. That is, for $m \in M$ and $r \in R$,

$$
m r=m \triangleleft s(r) \quad \text { and } \quad r m=m \triangleleft \mathrm{t}(r),
$$

where $r m$ denotes the left $R$-action on the left $\mathcal{K}$-comodule $M$ and $m r$ is the canonical right action defined in (2.3), i.e., $m r=\varepsilon\left(m_{\langle-1\rangle} \mathfrak{s}(r)\right) m_{\langle 0\rangle}$.

ii) For $k \in \mathcal{K}$ and $m \in M$,

$$
(m \triangleleft k)_{\langle-1\rangle} \otimes(m \triangleleft k)_{\langle 0\rangle}=k^{(2)^{+}} m_{\langle-1\rangle} k^{(1)} \otimes_{R} m_{\langle 0\rangle} \triangleleft k^{(2)^{-}} .
$$

The anti-Yetter-Drinfeld module $M$ is said to be stable if in addition, for any $m \in M$, $m_{\langle 0\rangle} m_{\langle-1\rangle}=m$.

Similarly one defines a left-right AYD module over a right $\times_{R}$-Hopf algebra $\mathscr{B}$ by a left $\mathscr{B}$-module and right $\mathscr{B}$-comodule $M$ satisfying:

i) $m r=\mathrm{t}(r) \triangleright m$ and $r m=\mathfrak{s}(r) \triangleright m$, where $m r$ denotes the right $R$-action on the right $\mathscr{B}$-comodule $M$ and $r m=m_{\langle 0\rangle} \cdot \varepsilon\left(\mathfrak{s}(r) m_{\langle 1\rangle}\right)$ is the canonical left $R$-action.

ii) For $b \in \mathscr{B}$ and $m \in M$,

$$
(b \triangleright m)_{\langle 0\rangle} \otimes(b \triangleright m)_{\langle 1\rangle}=b^{(1)^{+}} \triangleright m_{\langle 0\rangle} \otimes_{R} b^{(2)} m_{\langle 1\rangle} b^{(1)^{-}} .
$$

The anti-Yetter-Drinfeld module $M$ is said to be stable if in addition, for any $m \in M$, we have $m_{\langle 1\rangle} m_{\langle 0\rangle}=m$.

Remark 2.4. Left or right $\times_{R}$-Hopf algebras extend the notion of Hopf algebras. In fact if $\mathcal{K}$ is a bialgebra over a commutative ring $R$, with coproduct $k \mapsto k^{(1)} \otimes_{R} k^{(2)}$, then the bijectivity of the map $v$ is equivalent to the fact that $\mathcal{K}$ is a Hopf algebra. In this case the inverse of the map $v$ can be defined as

$$
v^{-1}(k \otimes 1)=k^{-} \otimes_{R^{\text {op }}} k^{+}:=k^{(1)} \otimes_{R^{\text {op }}} S\left(k^{(2)}\right),
$$


where $S$ denotes the antipode of the Hopf algebra $\mathcal{K}$. Therefore condition (2.5) for a left $\times_{R}$-Hopf algebra is equivalent to the relation

$$
(m k)_{\langle-1\rangle} \otimes(m k)_{\langle 0\rangle}=S\left(k^{(3)}\right) m_{\langle-1\rangle} k^{(1)} \otimes m_{\langle 0\rangle} k^{(2)} .
$$

Here we recall the definition of a character for $\times_{R}$-Hopf algebras. A map $\delta$ is called a right character [B], Lemma 2.5, for the $\times_{R}$-Hopf algebra $\mathcal{K}$ if it satisfies the following conditions:

$$
\begin{aligned}
\delta(k \mathfrak{s}(r)) & =\delta(k) r & & \text { for } k \in \mathcal{K} \text { and } r \in R, \\
\delta\left(k_{1} k_{2}\right) & =\delta\left(\mathfrak{s}\left(\delta\left(k_{1}\right)\right) k_{2}\right) & & \text { for } k_{1}, k_{2} \in \mathcal{K}, \\
\delta\left(1_{\mathcal{K}}\right) & =1_{R} . & &
\end{aligned}
$$

As an example, for any right $\times_{R}$-Hopf algebra the counit $\varepsilon$ is a right character. The following example is similar to [BS], Example 2.18, for right $\times_{R}$-Hopf algebras.

Example 2.5. Let $\mathcal{K}$ be a left $\times_{R}$-Hopf algebra, $\sigma \in \mathcal{K}$ a group-like element and the map $\delta: \mathcal{K} \rightarrow R$ a right character. The following action and coaction,

$$
r \triangleleft k=\delta(\mathfrak{s}(r) k) \quad \text { and } \quad r \mapsto \mathfrak{s}(r) \sigma \otimes 1,
$$

define a right $\mathcal{K}$-module and left $\mathcal{K}$-comodule structure on $R$, respectively. This action and coaction amount to a right-left anti-Yetter-Drinfeld module on $R$ if and only if, for all $r \in R$ and $k \in \mathcal{K}$,

$$
\mathfrak{s}(\delta(k)) \sigma=\mathrm{t}\left(\delta\left(k^{(2)-}\right)\right) k^{(2)+} \sigma k^{(1)} \quad \text { and } \quad \varepsilon(\sigma s(r))=\delta(\mathfrak{s}(r)) .
$$

The anti-Yetter-Drinfeld module $R$ is stable if in addition $\delta(\mathfrak{s}(r) \sigma)=r$ for all $r \in R$.

We specialize this example to the left $\times_{R}$-Hopf algebra $\mathcal{K}=R \otimes R^{\text {op }}$ explained in Example 2.1. To this end, we like to know all group-like elements and right characters of $\mathcal{K}$. One can easily characterize all homogenous group-like elements of $\mathcal{K}$. An element $x \otimes y \in \mathcal{K}$ is a group-like element if and only if $x y=y x=1$ because

$$
\begin{aligned}
\Delta(x \otimes y) & =(x \otimes y) \otimes_{R}(x \otimes y) \\
& =(x \otimes y) \otimes_{R}(x \otimes 1)(1 \otimes y) \\
& =(1 \otimes x)(x \otimes y) \otimes_{R}(1 \otimes y) \\
& =(x \otimes y x) \otimes_{R}(1 \otimes y) .
\end{aligned}
$$

On the other hand, one sees that $\Delta(x \otimes y)=(x \otimes 1) \otimes_{R}(1 \otimes y)$. Therefore $y x=1$. Since $x \otimes y$ is a group-like element, we have $1=\varepsilon(x \otimes y)=x y$. Conversely one easily sees that if $x y=y x=1$ then $x \otimes y$ is a group-like element for $\mathcal{K}$.

We claim that a map $\delta: \mathcal{K} \rightarrow R$ is a right character if and only if $\delta(s \otimes r)=\theta(s) t$ for some algebra map $\theta: R \rightarrow R$. Indeed, let $\delta$ be a right character on $\mathcal{K}$. Then

$$
\delta(s \otimes r)=\delta((1 \otimes r)(s \otimes 1))=\delta((1 \otimes r) \mathfrak{s}(s))=\delta(1 \otimes r) s .
$$


We define $\theta$ by $\theta(r):=\delta(1 \otimes r)$. It is obvious that $\theta$ is unital. We show that $\theta$ is an algebra map:

$$
\begin{aligned}
\theta\left(r r^{\prime}\right) & =\delta\left(1 \otimes r r^{\prime}\right) \\
& =\delta\left(\left(1 \otimes r^{\prime}\right)(1 \otimes r)\right) \\
& =\delta\left(\mathfrak{s}\left(\delta\left(\left(1 \otimes r^{\prime}\right)\right)(1 \otimes r)\right)\right. \\
& =\delta\left(( 1 \otimes r ) \mathfrak { s } \left(\delta\left(\left(1 \otimes r^{\prime}\right)\right)\right.\right. \\
& =\delta(1 \otimes r) \delta\left(r^{\prime} \otimes 1\right) \\
& =\theta(r) \theta\left(r^{\prime}\right) .
\end{aligned}
$$

Conversely, let $\theta$ be an algebra endomorphism of $R$. We consider the map $\delta: \mathcal{K} \rightarrow R$,

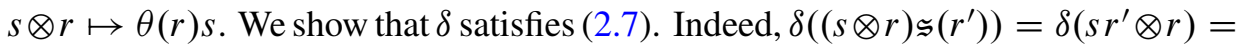
$\theta(r) s r^{\prime}=\delta(s \otimes r) r^{\prime}$. That $\delta$ satisfies (2.8) follows from

$$
\begin{aligned}
\delta\left((s \otimes r)\left(s^{\prime} \otimes r^{\prime}\right)\right) & =\delta\left(s s^{\prime} \otimes r^{\prime} r\right) \\
& =\theta\left(r^{\prime} r\right) s s^{\prime} \\
& =\theta\left(r^{\prime}\right) \theta(r) s s^{\prime} \\
& \left.=\delta\left(\theta(r) s s^{\prime} \otimes r^{\prime}\right)\right) \\
& =\delta\left((\theta(r) s \otimes 1)\left(s^{\prime} \otimes r^{\prime}\right)\right) \\
& =\delta\left(\left(\mathfrak{s}(\theta(r) s)\left(s^{\prime}, r^{\prime}\right)\right.\right. \\
& \left.=\delta\left(\mathfrak{s}(\delta(s, r))\left(s^{\prime} \otimes r^{\prime}\right)\right)\right) .
\end{aligned}
$$

Also since $\delta\left(1_{R} \otimes 1_{R}\right)=\theta\left(1_{R}\right) 1_{R}=1_{R}$, the map $\delta$ satisfies (2.9). Therefore $\delta$ is a right character.

Proposition 2.6. Let $\mathcal{K}$ be the left $\times_{R^{-H o p f}}$ algebra $R \otimes R^{\mathrm{op}}, x \otimes x^{-1} \in \mathcal{K} a$ homogenous group-like element and $\delta$ a right character on $\mathcal{K}$. Then the action and coaction defined in (2.10) amount to a right-left SAYD module on $R$ if and only if $x$ belongs the center of the algebra $R$ and $\theta=\mathrm{Id}$.

Proof. Let $k \otimes t \in \mathcal{K}$ and $r \in R$. By the characterization of all right characters on $\mathcal{K}$ the action and coaction defined in (2.10) reduce to

$$
r \triangleleft(k \otimes t)=\theta(t) r k \quad \text { and } \quad r \mapsto\left(r x \otimes x^{-1}\right) \otimes 1 .
$$

Now we show that $x$ is in the center of the algebra $R$. For this we use the stability condition

$$
r=\delta\left(\mathfrak{s}(r)\left(x \otimes x^{-1}\right)\right)=\delta\left((r \otimes 1)\left(x \otimes x^{-1}\right)\right)=\delta\left(r x \otimes x^{-1}\right) \quad \text { for all } r \in R .
$$

In the previous equality let $r=x^{-1}$. We have

$$
y=\delta\left(1 \otimes x^{-1}\right)=\theta\left(x^{-1}\right) .
$$


On the other hand,

$$
r=\delta\left(\mathfrak{s}(r)\left(x \otimes x^{-1}\right)\right)=\delta\left(r x \otimes x^{-1}\right)=\theta\left(x^{-1}\right) r x
$$

By multiplying the both sides by $x^{-1}$, we obtain $\theta\left(x^{-1}\right) r=r x^{-1}$. Therefore $x^{-1} r=r x^{-1}$ for all $r \in R$. Thus $x$ is also in the center of the algebra $R$.

We shall show that the AYD condition implies that the map $\theta$ is the identity map. The AYD condition is equivalent to

$$
\mathfrak{s}(\delta(k)) \sigma=t\left(\delta\left(k^{(2)^{-}}\right)\right) k^{(2)^{+}} \sigma k^{(1)} .
$$

Let $k=g \otimes h$ and $\sigma=x \otimes x^{-1}$. We have

$$
\theta(h) g x \otimes x^{-1}=(h \otimes 1)\left(x \otimes x^{-1}\right)(g \otimes 1)=h x g \otimes x^{-1},
$$

and therefore $\theta(h) g x=h x g$. Since $x$ is in the center of $R, \theta(h) g x=h g x$. By multiplying both sides by $x^{-1}$ from the right, we obtain $\theta(h) g=h g$, and for $g=1$ we have $\theta(h)=h$ for all $h \in R$. Thus we obtain the claimed action and coaction.

Conversely, it is easy to check that if $x$ belongs the center of $R$ and $\theta=\mathrm{Id}$, then the following $R \otimes R^{\mathrm{op}}$-action and coaction,

$$
r \triangleleft(k \otimes t)=t r k \quad \text { and } \quad r \mapsto\left(r x \otimes x^{-1}\right) \otimes 1, \quad k, t, r \in R,
$$

define a right-left SAYD module $R$ over the left $\times_{R}$-Hopf algebra $R \otimes R^{\text {op }}$.

2.3. Cyclic homology of module algebras and module corings. In this subsection we recall from $[\mathrm{BS}]$ the cyclic homology of module algebras and module corings with coefficients in SAYD modules under the symmetry of $\times_{R}$-Hopf algebras . In the case of module algebras, first we introduce a cocyclic module for left $\times_{R}$-Hopf algebras and then we dualize it, as explained in Section 1 to find a cyclic module.

Let $R$ be an algebra over $\mathbb{C}$ and $\mathcal{K}$ be a left $\times_{R}$-Hopf algebra. A left $\mathcal{K}$-module algebra $A$ [BS], Definition 2.3, is a $\mathbb{C}$-algebra and a left $\mathcal{K}$-module such that for all $k \in \mathcal{K}, a, a^{\prime} \in A$ and $r \in R$, the following identities hold:

$$
\begin{aligned}
k \triangleright 1_{A} & =\mathfrak{s}(\varepsilon(k)) \triangleright 1_{A}, \\
k \triangleright\left(a a^{\prime}\right) & =\left(k^{(1)} \triangleright a\right)\left(k^{(2)} \triangleright a^{\prime}\right), \\
(\mathrm{t}(r) \triangleright a) a^{\prime} & =a\left(\mathfrak{s}(r) \triangleright a^{\prime}\right) \quad \text { (multiplication is } R \text {-balanced). }
\end{aligned}
$$

Proposition 2.7. Let $S$ be an algebra over $\mathbb{C}, \mathcal{K}$ a left $\times_{S}$-Hopf algebra, $T$ a left $\mathcal{K}$-module algebra, and $M$ a right-left $S A Y D$ module over $\mathcal{K}$. Let

$$
{ }_{\mathcal{K}} C^{n}(T, M)=M \otimes \mathcal{K} T^{\otimes_{S}(n+1)} .
$$


Then the following cofaces, codegeneracies and cyclic map define a cocyclic module structure on ${ }_{\mathcal{K}} C^{*}(T, M)$ :

$$
\begin{aligned}
d_{0}(m \otimes \mathcal{K} \tilde{t}) & =m \otimes_{\mathcal{K}} 1 \otimes_{S} t_{0} \otimes_{S} \cdots \otimes_{S} t_{n}, \\
d_{i}(m \otimes \mathcal{K} \tilde{t}) & =m \otimes_{\mathcal{K}} t_{0} \otimes_{S} \cdots \otimes_{S} t_{i} \otimes_{S} 1 \otimes_{S} t_{i+1} \otimes \cdots \otimes_{S} t_{n}, \quad 1 \leq i \leq n, \\
d_{n}(m \otimes \mathcal{K} \tilde{t}) & =m_{\langle 0\rangle} \otimes_{\mathcal{K}} t_{0} \otimes_{S} \cdots \otimes_{S} t_{n} \otimes_{S} m_{\langle-1\rangle} \triangleright 1_{T}, \\
s_{i}(m \otimes \mathcal{K} \tilde{t}) & =m \otimes_{\mathcal{K}} t_{0} \otimes_{S} \cdots \otimes_{S} t_{i} t_{i+1} \otimes_{S} \ldots t_{n}, \quad 0 \leq i \leq n-1, \\
\mathrm{t}_{n}(m \otimes \mathcal{K} \tilde{t}) & =m_{\langle 0\rangle} \otimes_{\mathcal{K}} t_{1} \otimes_{S} \cdots \otimes_{S} t_{n} \otimes_{S} m_{\langle-1\rangle} \triangleright t_{0} .
\end{aligned}
$$

Here $\tilde{t}$ stands for $t_{0} \otimes_{S} \cdots \otimes_{S} t_{n}$.

Proof. Since $T$ is an $\mathcal{K}$-module algebra, by the $S$-balanced property of $T$, we have $k \triangleright 1_{T}=\mathfrak{s}(\varepsilon(k)) 1_{T}=\mathrm{t}(\varepsilon(k)) 1_{T}$. Therefore, cofaces are well defined. Here we consider $T^{\otimes_{S}(n+1)}$ as a $\mathcal{K}$-module with diagonal action. By (2.12) the codegeneracies are well defined. We show that the cocyclic map is well defined:

$$
\begin{aligned}
\mathrm{t}_{n} & \left(m \triangleleft k \otimes_{\mathcal{K}} t_{0} \otimes_{S} \cdots \otimes_{S} t_{n}\right) \\
& =(m \triangleleft k)_{\langle 0\rangle} \otimes_{\mathcal{K}} t_{1} \otimes_{S} \cdots \otimes_{S} t_{n} \otimes_{S}\left((m \triangleleft k)_{\langle-1\rangle} \triangleright t_{0}\right. \\
& =m_{\langle 0\rangle} \triangleleft k^{(2)^{-}} \otimes_{\mathcal{K}} t_{1} \otimes_{S} \cdots \otimes_{S} t_{n} \otimes_{S} k^{(2)}{ }^{+} m_{\langle 0\rangle} k^{(1)} \triangleright t_{0} \\
& =m_{\langle 0\rangle} \triangleleft k^{-(2)} \otimes_{\mathcal{K}} t_{1} \otimes_{S} \cdots \otimes_{S} t_{n} \otimes_{S} k^{+} m_{\langle-1\rangle} k^{-(1)} \triangleright t_{0} \\
& =m_{\langle 0\rangle} \otimes_{\mathcal{K}} k^{-(2)} \triangleright\left(t_{1} \otimes_{S} \cdots \otimes_{S} t_{n} \otimes_{S} k^{+} m_{\langle-1\rangle} k^{-(1)} \triangleright t_{0}\right) \\
& =m_{\langle 0\rangle} \otimes_{\mathcal{K}} k^{-(2)(1)} \triangleright t_{1} \otimes_{S} \cdots \otimes_{S} k^{-(2)(n)} \triangleright t_{n} \otimes_{S} \\
& \quad k^{-(2)(n+1)} k^{+} m_{\langle-1\rangle} k^{-(1)} \triangleright t_{0} \\
& =m_{\langle 0\rangle} \otimes_{\mathcal{K}} k^{-(2)} \triangleright t_{1} \otimes_{S} \cdots \otimes_{S} k^{-(n+1)} \triangleright t_{n} \otimes_{S} k^{-(n+2)} k^{+} m_{\langle-1\rangle} k^{-(1)} \triangleright t_{0} \\
& =m_{\langle 0\rangle} \otimes_{\mathcal{K}} k^{(2)} \triangleright t_{1} \otimes_{S} \cdots \otimes_{S} k^{(n+1)} \triangleright t_{n} \otimes_{S} m_{\langle-1\rangle} k^{(1)} \triangleright t_{0} \\
& =\mathrm{t}_{n}\left(m \otimes \mathcal{K} k^{(1)} \triangleright t_{0} \otimes_{S} \cdots \otimes_{S} k^{(n+1)} \triangleright t_{n}\right) \\
& =\mathrm{t}_{n}\left(m \otimes \mathcal{K} k \triangleright\left(t_{0} \otimes_{S} \cdots \otimes_{S} t_{n}\right)\right) .
\end{aligned}
$$

In the above, we use the SAYD condition (2.5) in the second equality, Proposition $2.2(\mathrm{v})$ in the third equality, the diagonal action of $\mathcal{K}$ on $T^{\otimes_{S}(n+1)}$ in the fifth equality and Proposition 2.2 (i) in the seventh equality. Now we check some of the cocyclicity conditions. To see that $\mathrm{t}_{n}^{n+1}=\mathrm{Id}$, we have

$$
\begin{aligned}
& \mathrm{t}_{n}^{n+1}\left(m \otimes \mathcal{K} t_{0} \otimes_{S} \cdots \otimes_{S} t_{n+1}\right)=m_{\langle 0\rangle} \otimes_{\mathcal{K}} m_{\langle-n-1\rangle} t_{0} \otimes_{S} \cdots \otimes_{S} m_{\langle-1\rangle} t_{n} \\
& =m_{\langle 0\rangle} \otimes \mathcal{K} m_{\langle-1\rangle} \triangleright\left(t_{0} \otimes_{S} \cdots \otimes_{S} t_{n}\right) \\
& =m_{\langle 0\rangle} m_{\langle-1\rangle} \otimes \mathcal{K} t_{0} \otimes_{S} \cdots \otimes_{S} t_{n} \\
& =m \otimes \mathcal{K} t_{0} \otimes_{S} \cdots \otimes_{S} t_{n} \text {. }
\end{aligned}
$$

In the above, we use the left diagonal action of $\mathcal{K}$ on $T^{\otimes_{S}(n+1)}$ in the second equality and the stability condition in the last equality. 
Now we show that $\mathrm{t}_{n} s_{0}=s_{n} \mathrm{t}_{n+1}^{2}$.

$$
\begin{aligned}
& s_{n} \mathrm{t}_{n+1}^{2}\left(m \otimes \mathcal{K} t_{0} \otimes_{S} \cdots \otimes_{S} t_{n+1}\right) \\
& \quad=s_{n} \mathrm{t}_{n+1}\left(m_{\langle 0\rangle} \otimes_{\mathcal{K}} t_{1} \otimes_{S} \cdots \otimes_{S} t_{n+1} \otimes_{S} m_{\langle-1\rangle} \triangleright t_{0}\right) \\
& \quad=s_{n}\left(m_{\langle 0\rangle\langle 0\rangle} \otimes_{\mathcal{K}} t_{2} \otimes_{S} \cdots \otimes_{S} t_{n+1} \otimes_{S} m_{\langle-1\rangle} \triangleright t_{0} \otimes_{S} m_{\langle 0\rangle\langle-1\rangle} \triangleright t_{1}\right) \\
& \quad=s_{n}\left(m_{\langle 0\rangle} \otimes_{\mathcal{K}} t_{2} \otimes_{S} \cdots \otimes_{S} t_{n+1} \otimes_{S} m_{\langle-1\rangle}^{(1)} t_{0} \otimes_{S} m_{\langle-1\rangle}^{(2)} \triangleright t_{1}\right) \\
& \quad=m_{\langle 0\rangle} \otimes_{\mathcal{K}} t_{2} \otimes_{S} \cdots \otimes_{S} t_{n+1} \otimes_{S}\left(m_{\langle-1\rangle}^{(1)} \triangleright t_{0}\right)\left(m_{\langle-1\rangle}^{(2)} \triangleright t_{1}\right) \\
& \quad=m_{\langle 0\rangle} \otimes_{\mathcal{K}} t_{2} \otimes_{S} \cdots \otimes_{S} t_{n+1} \otimes_{S} m_{\langle-1\rangle} \triangleright\left(t_{0} t_{1}\right) \\
& \quad=\mathrm{t}_{n}\left(m \otimes \mathcal{K} t_{0} t_{1} \otimes_{S} t_{2} \otimes_{S} \cdots \otimes_{S} t_{n+1}\right) \mathrm{t}_{n} s_{0}\left(m \otimes_{\mathcal{K}} t_{0} \otimes_{S} \cdots \otimes_{S} t_{n+1}\right) .
\end{aligned}
$$

In the above, we use the left $\mathcal{K}$-coaction property of $M$ in the third equality and left $\mathcal{K}$-module algebra property of $T$ in the fifth equality. The relation $t_{n} d_{0}=d_{n}$ is obvious. The other cocyclicity conditions can be easily verified.

The cyclic cohomology of the preceding cocyclic module, which is denoted by ${ }_{\mathcal{K}} \mathrm{HC}^{*}(T, M)$, is called Hopf cyclic cohomology of $T$ with coefficients in $M$ under the symmetry of $\mathcal{K}$.

To get a cyclic module we apply the duality procedure to the previous cocyclic module as follows. Let $S$ be an algebra over $\mathbb{C}, \mathcal{K}$ a left $\times{ }_{S}$-Hopf algebra, $T$ a left $\mathcal{K}$-module algebra and $M$ a right-left SAYD module over $\mathcal{K}$. Let

$$
{ }_{\mathcal{K}} C_{n}(T, M)=M \otimes \mathcal{K} T^{\otimes_{S}(n+1)} .
$$

The following faces, degeneracies and cyclic map define a cyclic module structure on $\mathcal{K} C_{*}(T, M)$ :

$$
\begin{aligned}
\delta_{i}\left(m \otimes_{\mathcal{K}} \tilde{t}\right) & =m \otimes_{\mathcal{K}} t_{0} \otimes_{S} \cdots \otimes_{S} t_{i} t_{i+1} \otimes_{S} \cdots \otimes_{S} t_{n}, \quad 0 \leq i \leq n-1, \\
\delta_{n}\left(m \otimes_{\mathcal{K}} \tilde{t}\right) & =m_{\langle 0\rangle} \otimes_{\mathcal{K}} t_{n}\left(m_{\langle-n\rangle} \triangleright t_{0}\right) \otimes_{S} m_{\langle-n+1\rangle} t_{1} \otimes_{S} \cdots \otimes_{S} m_{\langle-1\rangle} \triangleright t_{n-1}, \\
\sigma_{i}\left(m \otimes_{\mathcal{K}} \tilde{t}\right) & =m \otimes_{\mathcal{K}} t_{0} \otimes_{S} \cdots \otimes_{S} t_{i} \otimes_{S} 1 \otimes_{S} t_{i+1} \otimes_{S} \cdots \otimes_{S} t_{n}, \quad 0 \leq i \leq n, \\
\tau_{n}\left(m \otimes_{\mathcal{K}} \tilde{t}\right) & =m_{\langle 0\rangle} \otimes_{\mathcal{K}} t_{n} \otimes_{S} m_{\langle-n\rangle} \triangleright t_{0} \otimes_{S} \cdots \otimes_{S} m_{\langle-1\rangle} \triangleright t_{n-1} .
\end{aligned}
$$

Now we recall the cyclic homology of a module coring with coefficients in a SAYD module under the symmetry of a $\times_{R}$-Hopf algebra. Let $\mathscr{B}$ be a right $\times_{R^{-}}$ Hopf algebra. A right $\mathcal{B}$-module coring $C$ is an $R$-coring and right $\mathcal{B}$-module such that $R$-bimodule structure of $C$ coincides with the one induced by $\mathscr{B}$. In addition, it is assumed that the counit $\varepsilon$ and comultiplication $\Delta$ are right $\mathscr{B}$ linear. That is we consider the right $\mathscr{B}$-module structure of $R$ by $r \triangleleft b:=\varepsilon(\mathfrak{s}(r) b)$ and right $\mathscr{B}$ module structure of $C \otimes_{R} C$ is by the diagonal action. This means:

$$
\begin{gathered}
\varepsilon_{C}(c \triangleleft b)=\varepsilon_{C}(c) \triangleleft b=\varepsilon_{\mathcal{B}}\left(\mathfrak{s}\left(\varepsilon_{C}(c)\right) b\right), \\
\Delta_{C}(c \triangleleft b)=\Delta(c) \triangleleft b=c^{(1)} \triangleleft b^{(1)} \otimes_{R} c^{(2)} \triangleleft b^{(2)} .
\end{gathered}
$$


We define

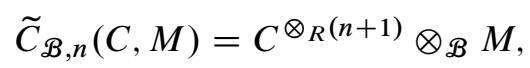

and following operators on $\widetilde{C}_{\mathscr{B}, n}(C, M)$ :

$$
\begin{aligned}
& \delta_{i}\left(\tilde{c} \otimes_{\mathcal{B}} m\right)=c_{0} \otimes_{R} \cdots \otimes_{R} \varepsilon_{C}\left(c_{i}\right) \otimes_{R} \cdots \otimes_{R} c_{n} \otimes_{\mathcal{B}} m, \quad 0 \leq i \leq n, \\
& \sigma_{i}\left(\tilde{c} \otimes_{\mathcal{B}} m\right)=c_{0} \otimes_{R} \cdots \otimes_{R} \Delta_{C}\left(c_{i}\right) \otimes_{R} \cdots \otimes_{R} c_{n} \otimes_{\mathcal{B}} m, \quad 0 \leq i \leq n, \\
& \tau_{n}\left(\tilde{c} \otimes_{\mathcal{B}} m\right)=c_{n} \triangleleft m_{\langle 1\rangle} \otimes_{R} c_{0} \otimes_{R} \cdots \otimes_{R} c_{n-1} \otimes_{\mathcal{B}} m_{\langle 0\rangle} .
\end{aligned}
$$

Here $\tilde{c}=c_{0} \otimes_{R} \cdots \otimes_{R} c_{n}$ and $C^{\otimes_{R}(n+1)}$ is a right $\mathcal{B}$-module by diagonal action. After some identifications the following theorem coincides with Proposition 2.19 in [BS]. For the reader's convenience we just check that the cyclic operator is well defined.

Proposition 2.8. Let $\mathcal{B}$ be a right $\times_{R}$-Hopf algebra, $M$ a left-right SAYD module over $\mathcal{B}$ and $C$ a right $\mathcal{B}$-module coring. Then the operators defined in (2.15) define a cyclic module structure on $\widetilde{C}_{\mathscr{B}, *}(C, M)$.

Proof. Since $C$ is a right $\mathcal{B}$-module coring we have, $\left(c \triangleleft b^{(1)}\right)\left(r \triangleleft b^{(2)}\right)=(c r) \triangleleft b$, for $c \in C$ and $r \in R$. Therefore $\left(c_{1} \triangleleft b^{(1)}\right)\left(\varepsilon\left(c_{2}\right) \triangleleft b^{(2)}\right)=\left(c_{1} \varepsilon\left(c_{2}\right)\right) \triangleleft b$ for $c_{1}, c_{2} \in R$, shows that faces are well defined. Also since comultiplication is a right $\mathcal{B}$-module map, using (2.14) degeneracies are well defined. The following calculation shows that the cyclic map is well defined:

$$
\begin{aligned}
& \tau_{n}\left(c_{0} \otimes_{R} \cdots \otimes_{R} c_{n} \otimes_{B} b \triangleright m\right) \\
& =c_{n} \triangleleft(b \triangleright m)_{\langle 1\rangle} \otimes_{R} c_{0} \cdots \otimes_{R} c_{n-1} \otimes_{\mathcal{B}}(b \triangleright m)_{\langle 0\rangle} \\
& =c_{n} \triangleleft b^{(2)} m_{\langle 1\rangle} b^{(1)^{-}} \otimes_{R} c_{0} \otimes_{R} \cdots \otimes_{R} c_{n-1} \otimes_{\mathcal{B}} b^{(1)^{+}} \triangleright m_{\langle 0\rangle} \\
& =\left(\left(c_{n} \triangleleft b^{+(2)} m_{\langle 1\rangle} b^{-}\right) \otimes_{R} c_{0} \otimes_{R} \cdots \otimes_{R} c_{n-1}\right) \triangleleft b^{+(1)} \otimes_{\mathcal{B}} m_{\langle 0\rangle} \\
& =\left(\left(c_{n} \triangleleft b^{+(2)} m_{\langle 1\rangle} b^{-} b^{+(1)(1)}\right) \otimes_{R} c_{0} \triangleleft b^{+(1)(2)} \otimes_{R} \cdots\right. \\
& \cdots \otimes_{R} c_{n-1} \triangleleft b^{+(1)(n+1)} \otimes_{B} m_{\langle 0\rangle} \\
& =\left(\left(c_{n} \triangleleft b^{+{ }^{(n+2)}} m_{\langle 1\rangle} b^{-} b^{+(1)}\right) \otimes_{R} c_{0} \triangleleft b^{+(2)} \otimes_{R} \cdots\right. \\
& \cdots \otimes_{R} c_{n-1} \triangleleft b^{+(n+1)} \otimes_{B} m_{\langle 0\rangle} \\
& =\left(c_{n} \triangleleft b^{(n+1)} m_{\langle 1\rangle}\right) \otimes_{R} c_{0} \triangleleft b^{(1)} \otimes_{R} \cdots \otimes_{R} c_{n-1} \triangleleft b^{(n)} \otimes_{\mathcal{B}} m_{\langle 0\rangle} \\
& =\tau_{n}\left(c_{0} \triangleleft b^{(1)} \otimes_{R} \cdots \otimes_{R} \otimes_{R} c_{n} \triangleleft b^{(n+1)} \otimes_{\mathcal{B}} m\right) \\
& =\tau_{n}\left(\left(c_{0} \otimes_{R} \cdots \otimes_{R} c_{n}\right) \triangleleft b \otimes_{B} m\right) \text {. }
\end{aligned}
$$

We use the AYD condition (2.6) in the second equality, [BS], Lemma 2.14 (v), in the third equality, the diagonal action in the fourth equality and [BS], Lemma 2.14 (i), in the sixth equality. We leave to the reader to check that $\delta_{i}, \sigma_{j}$ and $\tau$ satisfy all conditions of a cyclic module. 
The cyclic homology of this cyclic module is denoted by $\widetilde{\mathrm{HC}}_{\mathscr{B}, *}(C, M)$.

In a special case, $\mathscr{B}$ is a right $\mathscr{B}$-module coring by multiplication map $\mathscr{B} \otimes_{R} \mathscr{B} \rightarrow$ $\mathscr{B}$. To synchronize with the Hopf cyclic complex of Hopf algebras we identify

$$
\widetilde{C}_{\mathcal{B}, n}(\mathcal{B}, M) \stackrel{\varphi_{n}}{\longrightarrow} \mathcal{B}^{\otimes_{R} n} \otimes_{R^{\text {op }}} M,
$$

where $\varphi$ defined by

$$
\begin{aligned}
& \varphi_{n}: \mathcal{B}^{\otimes_{R}(n+1)} \otimes_{\mathcal{B}} M \rightarrow \mathcal{B}^{\otimes_{R} n} \otimes_{R^{\text {op }}} M, \\
& \varphi_{n}\left(b_{0} \otimes_{R} \cdots \otimes_{R} b_{n} \otimes_{\mathcal{B}} m\right)=\left(b_{0} \otimes_{R} \cdots \otimes_{R} b_{n-1}\right) \triangleleft b_{n}^{-} \otimes_{R^{\text {op }}} b_{n}^{+} m .
\end{aligned}
$$

Proposition 2.9. The map $\varphi_{n}$ defined in (2.16) is a well-defined isomorphism of vector spaces.

Proof. By [BS], Lemma 2.14 (iii), (ii), the map $\varphi$ is well defined. We define the inverse of map $\varphi$ by

$$
\varphi_{n}^{-1}\left(b_{1} \otimes_{R} \cdots \otimes_{R} b_{n} \otimes_{R^{\text {op }}} m\right)=b_{1} \otimes_{R} \cdots \otimes_{R} b_{n} \otimes_{R} 1_{\mathcal{B}} \otimes_{\mathcal{B}} m .
$$

This map is well defined because for any $r^{\mathrm{o}} \in R^{\mathrm{op}}$ we have $r^{\mathrm{o}} m=\mathrm{t}(r) \triangleright m$ and $\left(b_{1} \otimes_{R} \cdots \otimes_{R} b_{n}\right) \triangleleft r^{0}=b_{1} \mathrm{t}(r) \otimes_{R} \cdots \otimes_{R} b_{n}$. One can easily check that $\varphi \varphi^{-1}=\mathrm{Id}$ by [BS], Lemma 2.14 (iv), and $\varphi^{-1} \varphi=$ Id by [BS], Lemma 2.14 (i).

Therefore we transfer the cyclic structure of $\widetilde{C}_{\mathcal{B}, *}(\mathscr{B}, M)$ to $\mathscr{B}^{\otimes_{R}} \otimes_{R^{\text {op }}} M$. The resulting operators are recorded below.

$$
\begin{aligned}
& \delta_{i}\left(\tilde{b} \otimes_{R^{\text {op }}} m\right)=b_{1} \otimes_{R} \cdots \otimes_{R} \varepsilon\left(b_{i+1}\right) \otimes_{R} \cdots \otimes_{R} b_{n} \otimes_{R^{\text {op }}} m, \quad 0 \leq i \leq n-1 \\
& \delta_{n}\left(\tilde{b} \otimes_{R^{\text {op }}} m\right)=\left(b_{1} \otimes_{R} \cdots \otimes_{R} b_{n-1}\right) \triangleleft b_{n}^{-} \otimes_{R^{\text {op }}} b_{n}^{+} m, \\
& \sigma_{i}\left(\tilde{b} \otimes_{R^{\text {op }}} m\right)=b_{1} \otimes_{R} \cdots \otimes_{R} \Delta\left(b_{i+1}\right) \otimes_{R} \cdots \otimes_{R} b_{n} \otimes_{R^{\text {op }}} m, \quad 0 \leq i \leq n-1, \\
& \sigma_{n}\left(\tilde{b} \otimes_{R^{\text {op }}} m\right)=b_{1} \otimes_{R} \cdots \otimes_{R} b_{n} \otimes_{R} 1_{\mathcal{B}} \otimes_{R^{\text {op }}} m, \\
& \tau_{n}\left(\tilde{b} \otimes_{R^{\text {op }}} m\right)=\left(m_{\langle 1\rangle} \otimes_{R} b_{1} \otimes_{R} \cdots \otimes_{R} b_{n-1}\right) \triangleleft b_{n}^{-} \otimes_{R^{\text {op }}} b_{n}^{+} m_{\langle 0\rangle},
\end{aligned}
$$

where $\tilde{b}=b_{1} \otimes_{R} \cdots \otimes_{R} b_{n}$.

\section{Equivariant Hopf Galois extensions}

In this section we first define equivariant Hopf Galois extensions. An equivariant Hopf Galois extension is a quadruple $(\mathcal{K}, \mathscr{B}, T, S)$ satisfying certain properties as stated in Definition 3.1. In Section 3.1 we show that any equivariant Hopf Galois extension $(\mathcal{K}, \mathscr{B}, T, S)$ defines a functor from the category of SAYD modules over $\mathcal{K}$ 
to the category of SAYD modules over $\mathcal{B}$ such that their Hopf cyclic complexes with corresponding coefficients are isomorphic. In Section 3.2 we introduce an example of equivariant Hopf Galois extension and explicitly illustrate the functor between the categories of SAYD modules.

Let $\mathscr{B}$ be a right $\times_{R}$-Hopf algebra. We say the algebra $T$ is a right $\mathscr{B}$-comodule algebra via $\mathbf{\nabla}: T \rightarrow T \otimes_{R} \mathscr{B}$ if $T$ is an $R$-bimodule and a right $B$-comodule satisfying the following conditions:

i) $\left(t_{1} r\right) t_{2}=t_{1}\left(r t_{2}\right)$ (multiplication in $T$ is $R$-balanced),

ii) $1_{T_{\langle 0\rangle}} \otimes_{R} 1_{T_{\langle 1\rangle}}=1_{T} \otimes_{R} 1_{\mathscr{B}}$,

iii) $\left(t t^{\prime}\right)_{\langle 0\rangle} \otimes_{R}\left(t t^{\prime}\right)_{\langle 1\rangle}=t_{\langle 0\rangle} t_{\langle 0\rangle}^{\prime} \otimes_{R} t_{\langle 1\rangle} t_{\langle 1\rangle}^{\prime}$.

The coinvariants subalgebra $S \subseteq T$ is defined by

$$
S:=T^{\mathscr{B}}=\left\{s \in T \mid \mathbf{\nabla}(s)=s \otimes_{R} 1_{\mathscr{B}}\right\} .
$$

Definition 3.1. Let $R$ be an algebra over $\mathbb{C}, \mathscr{B}$ a right $\times_{R}$-Hopf algebra, $T$ a right $\mathscr{B}$-comodule algebra, $S=T^{\mathscr{B}}, \mathcal{K}$ a left $\times_{S}$-Hopf algebra, and $T$ a left $\mathcal{K}$-module algebra. $T$ is called a $\mathcal{K}$-equivariant $\mathscr{B}$-Galois extension of $S$, if the canonical map

$$
\text { can: } T \otimes_{S} T \rightarrow T \otimes_{R} \mathscr{B}, \quad t^{\prime} \otimes_{S} t \mapsto t^{\prime} t_{\langle 0\rangle} \otimes_{R} t_{\langle 1\rangle},
$$

is bijective and the right coaction of $\mathscr{B}$ over $T$ is $\mathcal{K}$-equivariant, i.e.,

$$
(k t)_{\langle 0\rangle} \otimes_{R}(k t)_{\langle 1\rangle}=k t_{\langle 0\rangle} \otimes_{R} t_{\langle 1\rangle}, \quad k \in \mathcal{K}, t \in T .
$$

We denote a $\mathcal{K}$-equivariant $\mathcal{B}$-Galois extension described in Definition 3.1 by $\mathcal{K} T(S)^{\mathcal{B}}$. One observes that by $\mathcal{K}:=B \otimes B^{\text {op }}$ in the above definition we recover the ordinary Hopf Galois extension since for any $\mathcal{B}$-comodule algebra $T$ the Galois

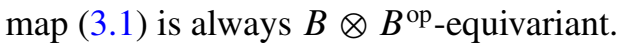

One defines left $\mathcal{K}$-module structures on $T \otimes_{S} T$ and $T \otimes_{R} \mathscr{B}$, respectively, as follows:

$$
k \triangleright\left(t_{1} \otimes_{S} t_{2}\right)=k^{(1)} t_{1} \otimes_{S} k^{(2)} t_{2},
$$

and

$$
k \triangleright\left(t \otimes_{R} b\right)=k t \otimes_{R} b .
$$

The $\mathcal{K}$-equivariant condition of $\mathcal{K} T(S)^{\mathcal{B}}$ implies that

$$
\operatorname{can}\left(k \triangleright\left(t^{\prime} \otimes_{S} t\right)\right)=k \triangleright \operatorname{can}\left(t^{\prime} \otimes_{S} t\right), \quad k \in \mathcal{K} t, t^{\prime} \in T .
$$

We denote the inverse of the Galois map (3.1) by the index notation

$$
\operatorname{can}^{-1}\left(1 \otimes_{R} b\right):=b_{\langle-\rangle} \otimes_{S} b_{\langle+\rangle} .
$$

One has the following properties for the maps can and $\mathrm{can}^{-1}$. 
Lemma 3.2. Let $\mathcal{K} T(S)^{\mathcal{B}}$ be a $\mathcal{K}$-equivariant $\mathcal{B}$-Galois extension. Then the following properties hold.
i) $(k \triangleright t) b_{\langle-\rangle} \otimes_{S} b_{\langle+\rangle}=\left(k^{(1)} t\right)\left(k^{(2)} b_{\langle-\rangle}\right) \otimes_{S} k^{(3)} b_{\langle+\rangle}$.
ii) $\left(b b^{\prime}\right)_{\langle-\rangle} \otimes_{S}\left(b b^{\prime}\right)_{\langle+\rangle}=b_{\langle-\rangle}^{\prime} b_{\langle-\rangle} \otimes_{S} b_{\langle+\rangle} b_{\langle+\rangle}^{\prime}$.
iii) $b_{\langle-\rangle\langle 0\rangle} \otimes_{R} b_{\langle-\rangle\langle 1\rangle} \otimes_{S} b_{\langle+\rangle}=b_{\langle-\rangle}^{+} \otimes_{R} b^{-} \otimes_{S} b_{\langle+\rangle}^{+}$.
iv) $b_{\langle-\rangle} \otimes_{S} b_{\langle+\rangle\langle 0\rangle} \otimes_{R} b_{\langle+\rangle\langle 1\rangle}=b_{\langle-\rangle}^{(1)} \otimes_{S} b_{\langle+\rangle}^{(1)} \otimes_{R} b^{(2)}$.
v) $b_{\langle-\rangle} b_{\langle+\rangle\langle 0\rangle} \otimes_{R} b_{\langle+\rangle\langle 1\rangle}=1 \otimes_{R} b$.
vi) $t_{\langle 0\rangle} t_{\langle 1\rangle\langle-\rangle} \otimes_{S} t_{\langle 1\rangle\langle+\rangle}=1 \otimes_{S} t$.
vii) $1_{\mathscr{B}\langle-\rangle} \otimes_{S} 1_{\mathcal{B}(+)}=1_{T} \otimes_{S} 1_{T}$.
viii) $\mathrm{t}(r)_{\langle-\rangle} \otimes_{S} \mathrm{t}(r)_{\langle+\rangle}=r \triangleright 1_{T} \otimes_{S} 1_{T}$.
ix) $\mathfrak{s}(r)_{\langle-\rangle} \otimes_{S} \mathfrak{s}(r)_{\langle+\rangle}=1_{T} \otimes_{S} 1_{T} \triangleleft r$.

Proof. One easily sees that (i) is equivalent to $\mathcal{K}$-equivariant property of the map $\mathrm{can}^{-1}$. One can find a proof of the rest in [BS], pp. 268-270.

3.1. Equivariant Hopf Galois extension as a functor. Let $\mathcal{K} T(S)^{\mathscr{B}}$ be a $\mathcal{K}$-equivariant $\mathscr{B}$-Galois extension, and $M$ be a right-left SAYD module over $\mathcal{K}$. We let $\mathscr{B}$ act on $\widetilde{M}:=M \otimes \mathcal{K} T$ on the left by

$$
b \triangleright(m \otimes \mathcal{K} t)=m_{\langle 0\rangle} \otimes \mathcal{K} b_{\langle+\rangle}\left(m_{\langle-1\rangle} \triangleright\left(t b_{\langle-\rangle}\right)\right) .
$$

We also let $\mathcal{B}$ coact on $\tilde{M}$ from the right by

$$
(m \otimes \mathcal{K} t) \mapsto m \otimes \mathcal{K}_{\langle 0\rangle} \otimes_{R} t_{\langle 1\rangle} .
$$

Theorem 3.3. Let $R$ be an algebra over $\mathbb{C}, \mathscr{B}$ a right $\times_{R}$-Hopf algebra, $T$ a right $\mathscr{B}$-comodule algebra, $S=T^{\mathcal{B}}, \mathcal{K}$ a left $\times_{S}$-Hopf algebra, $T$ a left $\mathcal{K}$-module algebra and $M$ be a right-left $S A Y D$ module over $\mathcal{K}$. If $\mathcal{K} T(S)^{\mathcal{B}}$ is a $\mathcal{K}$-equivariant $\mathscr{B}$-Galois extension, then $\widetilde{M}:=M \otimes \mathcal{K} T$ is a left-right $S A Y D$ module over $B$ by the action and coaction defined in (3.2) and (3.3).

Proof. The above coaction is obviously well defined by the $\mathcal{K}$-equivariant property of the coaction of $\mathscr{B}$ over $T$. We shall show that the action (3.2) is well defined. We show that

$$
b \triangleright\left(m \triangleleft k \otimes_{\mathcal{K}} t\right)=b \triangleright(m \otimes \mathcal{K} k \triangleright t), \quad b \in \mathscr{B}, m \in M, k \in \mathcal{K}, t \in T .
$$


Indeed,

$$
\begin{aligned}
b \triangleright(m \triangleleft k \otimes \mathcal{K} t) & =(m k)_{\langle 0\rangle} \otimes \mathcal{K} b_{\langle+\rangle}\left((m k)_{\langle-1\rangle} \triangleright\left(t b_{\langle-\rangle}\right)\right) \\
& =m_{\langle 0\rangle} k^{(2)^{-}} \otimes_{\mathcal{K}} b_{\langle+\rangle}\left(k^{(2)^{+}} m_{\langle-1\rangle} k^{(1)} \triangleright\left(t b_{\langle-\rangle}\right)\right) \\
& =m_{\langle 0\rangle} \otimes \mathcal{K}^{(2)^{-}} \triangleright\left(b_{\langle+\rangle}\left(k^{(2)^{+}} m_{\langle-1\rangle} k^{(1)} \triangleright\left(t b_{\langle-\rangle}\right)\right)\right) \\
& \left.=m_{\langle 0\rangle} \otimes \mathcal{K}\left(k^{(2)^{-(1)}} b_{\langle+\rangle}\right)\left(k^{(2)^{-(2)}} k^{(2)^{+}} m_{\langle-1\rangle} k^{(1)} \triangleright\left(t b_{\langle-\rangle}\right)\right)\right) \\
& =m_{\langle 0\rangle} \otimes \mathcal{K}\left(k^{(2)} \triangleright b_{\langle+\rangle}\right)\left(m_{\langle-1\rangle} k^{(1)} \triangleright\left(t b_{\langle-\rangle}\right)\right) \\
& =m_{\langle 0\rangle} \otimes \mathcal{K}\left(k^{(3)} \triangleright b_{\langle+\rangle}\right)\left(m_{\langle-1\rangle} \triangleright\left(\left(k^{(1)} \triangleright t\right)\left(k^{(2)} \triangleright b_{\langle-\rangle}\right)\right)\right) \\
& =m_{\langle 0\rangle} \otimes \mathcal{K} b_{\langle+\rangle}\left(m_{\langle-1\rangle} \triangleright\left((k \triangleright t) b_{\langle-\rangle}\right)\right) \\
& =b \triangleright(m \otimes \mathcal{K} k \triangleright t) .
\end{aligned}
$$

We use the SAYD condition (2.5) for $M$ over $\mathcal{K}$ in the second equality, the $\mathcal{K}$-module algebra property of $T$ in the fourth equality, the Lemma 2.2 (i) in the fifth equality, the $\mathcal{K}$-module algebra property of $T$ in the sixth equality and the Lemma 3.2 (i) in the seventh equality. Next we show that the action (3.2) is $S$-balanced both in $b_{\langle-\rangle} \otimes_{S} b_{\langle+\rangle}$and $m_{\langle-1\rangle} \otimes_{S} m_{\langle 0\rangle}$. That is for any $q, p \in S, m \in M, k \in \mathcal{K}$ and $t_{1}, t_{2}, t_{3} \in T$, we should show

$$
p m \otimes \mathcal{K} t_{1}\left(k \triangleright t_{2} t_{3}\right)=m \otimes \mathcal{K} t_{1}\left(\mathrm{t}(p) k \triangleright t_{2} t_{3}\right),
$$

which is obvious because

$$
\begin{aligned}
p m \otimes \mathcal{K} t_{1}\left(k \triangleright t_{2} t_{3}\right) & =m \triangleleft \mathrm{t}(p) \otimes \mathcal{K} t_{1}\left(k \triangleright t_{2} t_{3}\right) \\
& =m \otimes \mathcal{K}(p) \triangleright t_{1}\left(k \triangleright t_{2} t_{3}\right) \\
& =m \otimes \mathcal{K} t_{1}\left(\mathrm{t}(p) k \triangleright t_{2} t_{3}\right),
\end{aligned}
$$

where $\mathrm{t}$ is the target map of the left bialgebroid $\mathcal{K}$. In the above we use $p m=m \triangleleft \mathrm{t}(p)$ and $\Delta(\mathrm{t}(p))=1 \mathcal{K} \otimes_{S} \mathrm{t}(p)$. This allows then to substitute $k \otimes_{S} m=n_{\langle-1\rangle} \otimes_{S} n_{\langle 0\rangle}$ for any $n \in M$. To finish the argument that the action (3.2) is $S$-balanced we should also show

$$
m_{\langle 0\rangle} \otimes \mathcal{K} t_{1}\left(m_{\langle-\rangle} \triangleright t_{2} t_{3} q\right)=m_{\langle 0\rangle} \otimes_{\mathcal{K}} q t_{1}\left(m_{\langle-\rangle} \triangleright t_{2} t_{3}\right),
$$

which is clear because

$$
\begin{aligned}
m_{\langle 0\rangle} \otimes \mathcal{K} t_{1}\left(m_{\langle-1\rangle} \triangleright t_{2} t_{3} q\right) & =m_{\langle 0\rangle} \otimes \mathcal{K} t_{1}\left(m_{\langle-1\rangle} \mathrm{t}(q) \triangleright t_{2} t_{3}\right) \\
& =m_{\langle 0\rangle} q \otimes \mathcal{K} t_{1}\left(m_{\langle-1\rangle} \triangleright t_{2} t_{3}\right) \\
& =m_{\langle 0\rangle} \triangleleft \mathfrak{s}(q) \otimes \mathcal{K} t_{1}\left(m_{\langle-1\rangle} \triangleright t_{2} t_{3}\right) \\
& =m_{\langle 0\rangle} \otimes \mathcal{K} \mathfrak{s}(q) \triangleright t_{1}\left(m_{\langle-1\rangle} \triangleright t_{2} t_{3}\right) \\
& =m_{\langle 0\rangle} \otimes \mathcal{K} q t_{1}\left(m_{\langle-1\rangle} \triangleright t_{2} t_{3}\right),
\end{aligned}
$$


where $s$ is the source map of the left bialgebroid $\mathcal{K}$. In the above we use (2.4) in the second equality. Thus it makes sense to substitute $t_{3} \otimes_{S} t_{1}=b_{\langle-\rangle} \otimes_{S} b_{\langle+\rangle}$. Next we show that (3.2) is associative. For this, let $g, h \in \mathscr{B}$ and $m \otimes \mathcal{K} t \in \tilde{M}$.

$$
\begin{aligned}
& g \triangleright\left(h \triangleright\left(m \otimes_{\mathcal{K}} t\right)\right) \\
& =g \triangleright\left(m_{\langle 0\rangle} \otimes_{\mathcal{K}} h_{\langle+\rangle}\left(m_{\langle-1\rangle} \triangleright\left(t h_{\langle-\rangle}\right)\right)\right) \\
& =m_{\langle 0\rangle\langle 0\rangle} \otimes \mathcal{K} g_{\langle+\rangle}\left\{m_{\langle 0\rangle\langle-1\rangle} \triangleright\left[h_{\langle+\rangle}\left(m_{\langle-1\rangle} \triangleright\left(t h_{\langle-\rangle}\right)\right) g_{\langle-\rangle}\right]\right\} \\
& =m_{\langle 0\rangle} \otimes \mathcal{K} g_{\langle+\rangle}\left\{m_{\langle-1\rangle}^{(2)} \triangleright\left[h_{\langle+\rangle}\left(m_{\langle-1\rangle}^{(1)} \triangleright\left(t h_{\langle-\rangle}\right)\right) g_{\langle-\rangle}\right]\right\} \\
& =m_{\langle 0\rangle} \otimes_{\mathcal{K}} g_{\langle+\rangle}\left\{m_{\langle-1\rangle}^{(2)(1)} \triangleright\left[h_{\langle+\rangle}\left(m_{\langle-1\rangle}^{(1)} \triangleright\left(t h_{\langle-\rangle}\right)\right)\right]\right\}\left(m_{\langle-1\rangle}^{(2)(2)} \triangleright g_{\langle-\rangle}\right) \\
& =m_{\langle 0\rangle} \otimes \mathcal{K} g_{\langle+\rangle}\left\{m_{\langle-1\rangle}^{(2)(1)} \triangleright\left[h_{\langle+\rangle}\left(m_{\langle-1\rangle}^{(1)(1)} \triangleright t\right)\left(m_{\langle-1\rangle}^{(1)(2)} \triangleright h_{\langle-\rangle}\right)\right]\right\}\left(m_{\langle-1\rangle}^{(2)(2)} \triangleright g_{\langle-\rangle}\right) \\
& =m_{\langle 0\rangle} \otimes \mathcal{K} g_{\langle+\rangle}\left\{m_{\langle-1\rangle}^{(3)} \triangleright\left[h_{\langle+\rangle}\left(m_{\langle-1\rangle}^{(1)} \triangleright t\right)\left(m_{\langle-1\rangle}^{(2)} \triangleright h_{\langle-\rangle}\right)\right]\right\}\left(m_{\langle-1\rangle}^{(4)} \triangleright g_{\langle-\rangle}\right) \\
& =m_{\langle 0\rangle} \otimes \mathcal{K} g_{\langle+\rangle}\left(m_{\langle-1\rangle}^{(3)} \triangleright h_{\langle+\rangle}\right)\left[\left(m_{\langle-1\rangle}^{(4)} \triangleright\left(m_{\langle-1\rangle}^{(1)} \triangleright t\right)\left(m_{\langle-1\rangle}^{(2)} \triangleright h_{\langle-\rangle}\right)\right]\left(m_{\langle-1\rangle}^{(5)} \triangleright g_{\langle-\rangle}\right)\right. \\
& =m_{\langle 0\rangle} \otimes \mathcal{K} g_{\langle+\rangle} h_{\langle+\rangle}\left[\left(m_{\langle-1\rangle}^{(2)} \triangleright\left(m_{\langle-1\rangle}^{(1)} \triangleright t\right) h_{\langle-\rangle}\right]\left(m_{\langle-1\rangle}^{(3)} \triangleright g_{\langle-\rangle}\right)\right. \\
& \left.=m_{\langle 0\rangle} \otimes \mathcal{K} g_{\langle+\rangle} h_{\langle+\rangle}\left[m_{\langle-1\rangle}^{(2)} \triangleright\left(m_{\langle-1\rangle}^{(1)} \triangleright t\right) h_{\langle-\rangle} g_{\langle-\rangle}\right)\right] \\
& =m_{\langle 0\rangle\langle 0\rangle} \otimes_{\mathcal{K}}(g h)_{\langle+\rangle}\left[m_{\langle 0\rangle\langle-1\rangle} \triangleright\left(m_{\langle-1\rangle} \triangleright t\right)(g h)_{\langle-\rangle}\right] \\
& =(g h) \triangleright\left(m_{\langle 0\rangle} \otimes_{\mathcal{K}} m_{\langle-1\rangle} \triangleright t\right) \\
& =(g h) \triangleright\left(m \otimes_{\mathcal{K}} t\right) .
\end{aligned}
$$

We apply the coaction property in the second equality, the $\mathcal{K}$-module algebra property of $T$ in the third, fourth, sixth and eighth equalities, the $\mathcal{K}$-equivariance property of Lemma (3.2) (i) in the seventh equality and the stability condition in the last equality. Also unitality of the action (3.2) is obvious by Lemma (3.2) (vii) and stability condition. Now we verify the AYD condition for $\widetilde{M}$ over $\mathscr{B}$. Let $b \in B, t \in T$ and $m \in M$.

$$
\begin{aligned}
(b & \left.\triangleright\left(m \otimes_{\mathcal{K}} t\right)\right)_{\langle 0\rangle} \otimes_{R}\left(b \triangleright\left(m \otimes_{\mathcal{K}} t\right)\right)_{\langle 1\rangle} \\
& =\left(m_{\langle 0\rangle} \otimes_{\mathcal{K}} b_{\langle+\rangle}\left(m_{\langle-1\rangle} \triangleright\left(t b_{\langle-1\rangle}\right)\right)\right)_{\langle 0\rangle} \otimes_{R}\left(m_{\langle 0\rangle} \otimes_{\mathcal{K}} b_{\langle+\rangle}\left(m_{\langle-1\rangle} \triangleright\left(t b_{\langle-\rangle}\right)\right)\right)_{\langle 1\rangle} \\
& =m_{\langle 0\rangle} b_{\langle+\rangle\langle 0\rangle}\left(m_{\langle-1\rangle} \triangleright\left(t_{\langle 0\rangle} b_{\langle-\rangle\langle 0\rangle}\right)\right) \otimes_{R} b_{\langle+\rangle\langle 1\rangle} t_{\langle 1\rangle} b_{\langle-\rangle\langle 1\rangle} \\
& =m_{\langle 0\rangle} b_{\langle+\rangle}^{(1)}\left(m_{\langle-1\rangle} \triangleright\left(t_{\langle 0\rangle} b_{\langle-\rangle\langle 0\rangle}^{(1)}\right) \otimes_{R} b^{(2)} t_{\langle 1\rangle} b_{\langle-\rangle\langle 1\rangle}^{(1)}\right. \\
& =m_{\langle 0\rangle} \otimes_{\mathcal{K}} b_{\langle+\rangle}^{(1)}\left(m_{\langle-1\rangle} \triangleright\left(t_{\langle 0\rangle} b_{\langle-\rangle}^{(1)}\right)\right) \otimes_{R} b^{(2)} t_{\langle 1\rangle} b^{(1)}- \\
& =b^{(1)^{+}} \triangleright\left(m \otimes_{\mathcal{K}} t\right)_{\langle 0\rangle} \otimes_{R} b^{(2)}\left(m \otimes_{\mathcal{K}} t\right)_{\langle 1\rangle} b^{(1)^{-}} .
\end{aligned}
$$

We use the $\mathcal{K}$-equivariant property of the coaction of $\mathscr{B}$ on $T$ and the $\mathscr{B}$-comodule algebra property of $T$ in the second equality, the Lemma 3.2 (iv) in the third equality and the Lemma 3.2 (iii) in the fourth equality. 
The stability condition of $\tilde{M}$ over $\mathscr{B}$ follows from

$$
\begin{aligned}
(m \otimes \mathcal{K} t)_{\langle 1\rangle}(m \otimes \mathcal{K} t)_{\langle 0\rangle} & =t_{\langle 1\rangle}\left(m \otimes_{\mathcal{K}} t_{\langle 0\rangle}\right) \\
& =m_{\langle 0\rangle} \otimes_{\mathcal{K}}\left(t_{\langle 1\rangle\langle+\rangle}\left(m_{\langle-1\rangle} \triangleright\left(t_{\langle 0\rangle} t_{\langle 1\rangle\langle-\rangle}\right)\right)\right) \\
& =m_{\langle 0\rangle} \otimes_{\mathcal{K}} t\left(m_{\langle-1\rangle} \triangleright 1_{T}\right) \\
& =m_{\langle 0\rangle} \otimes_{\mathcal{K}} t\left(\mathrm{t}\left(\varepsilon\left(m_{\langle-1\rangle}\right)\right) \triangleright 1_{T}\right) \\
& =m_{\langle 0\rangle} \otimes_{\mathcal{K}} \mathrm{t}\left(\varepsilon\left(m_{\langle-1\rangle}\right)\right) \triangleright t \\
& =m_{\langle 0\rangle} \triangleleft \mathrm{t}\left(\varepsilon\left(m_{\langle-1\rangle}\right)\right) \otimes_{\mathcal{K}} t \\
& =m \otimes_{\mathcal{K}} t
\end{aligned}
$$

Lemma 3.2 (vi) is applied in the third equality, the module algebra property in fourth equality and the counitality of the coaction on $M$ is used in the last equality.

Let us denote the category of SAYD modules over a left $\times$-Hopf algebra $\mathcal{K}$ by $\mathcal{K}_{\text {SAYD }} \mathcal{K}$. Its objects are all right-left SAYD modules over $\mathcal{K}$ and its morphisms are all $\mathcal{K}$-linear-colinear maps. Similarly one denotes by ${ }_{\mathcal{B}} \mathrm{SAYD}^{\mathcal{B}}$ the category of leftright SAYD modules of a right $\times$-Hopf algebra $B$. We see that Theorem 3.3 amounts to the object map of a functor $F$ from ${ }^{\mathcal{K}} \mathrm{SAYD}_{\mathcal{K}}$ to ${ }_{\mathcal{B}} \mathrm{SAYD}^{\mathcal{B}}$. Let $\phi: M \rightarrow N$ be a morphism in ${ }^{\mathcal{K}} \mathrm{SAYD}_{\mathcal{K}}$ we define $F(\phi)$ to be $\phi \otimes \mathcal{K} \mathrm{Id}_{T}$.

Proposition 3.4. The assignment $F:{ }^{\mathcal{K}} \mathrm{SAYD}_{\mathcal{K}} \rightarrow{ }_{B} \mathrm{SAYD}^{\mathcal{B}}$ defines a covariant functor.

Proof. Using Theorem 3.3 we see that $F$ is an object map. We now prove that it is also a morphism map. Let $\phi: M \rightarrow N$ be a morphism in ${ }^{\mathcal{K}} \mathrm{SAYD}_{\mathcal{K}}$. The colinearity of $F(\phi)$ is obvious. The following shows that $F(\phi)$ is also linear,

$$
\begin{aligned}
(\phi \otimes \mathrm{Id})(b \triangleright(m \otimes \mathcal{K} t)) & =(\phi \otimes \mathrm{Id})\left(m_{\langle 0\rangle} \otimes_{\mathcal{K}} b_{\langle+\rangle}\left(m_{\langle-1\rangle} \triangleright\left(t b_{\langle-\rangle}\right)\right)\right) \\
& =\phi\left(m_{\langle 0\rangle}\right) \otimes_{\mathcal{K}} b_{\langle+\rangle}\left(m_{\langle-1\rangle} \triangleright\left(t b_{\langle-\rangle}\right)\right) \\
& =\phi(m)_{\langle 0\rangle} \otimes_{\mathcal{K}} b_{\langle+\rangle}\left(\phi(m)_{\langle-1\rangle} \triangleright\left(t b_{\langle-\rangle}\right)\right) \\
& =b \triangleright(\phi(m) \otimes t)=b \triangleright((\phi \otimes \mathrm{Id})(m \otimes \mathcal{K} t)) .
\end{aligned}
$$

We use the comodule map property of $\phi$ in the third equality. The covariant property of the functor is obvious.

Let us recall that ${ }_{\mathcal{K}} C_{n}(T, M)=M \otimes_{\mathcal{K}} T^{\otimes_{S} n+1}$ and $C_{\mathcal{B}, n}(B, \tilde{M}):=B^{\otimes_{R} n} \otimes_{R^{\text {op }}}$ $\tilde{M}$ are the cyclic modules defined in (2.13) and (2.17) respectively. Now we define the maps

$$
\omega_{n}: M \otimes_{\mathcal{K}} T^{\otimes_{S}(n+1)} \rightarrow \mathcal{B}^{\otimes_{R} n} \otimes_{R^{\text {op }}} \tilde{M},
$$

given by

$$
\begin{array}{r}
\omega_{n}\left(m \otimes_{\mathcal{K}} t_{0} \otimes_{S} \cdots \otimes_{S} t_{n}\right)=t_{1\langle 1\rangle} t_{2\langle 1\rangle} \ldots t_{n\langle 1\rangle} \otimes_{R} t_{2\langle 2\rangle} \ldots t_{n\langle 2\rangle} \otimes_{R} \ldots \\
\cdots \otimes_{R} t_{n\langle n\rangle} \otimes_{R^{\mathrm{op}}}\left(m \otimes_{\mathcal{K}} t_{0} t_{1\langle 0\rangle} \ldots t_{n\langle 0\rangle}\right),
\end{array}
$$


and

$$
\begin{aligned}
& \omega_{n}^{-1}\left(b_{1} \otimes_{R} \cdots \otimes_{R} b_{n+1} \otimes_{R^{\mathrm{op}}} m \otimes_{K} t\right) \\
& =m \otimes_{K} t b_{1\langle-\rangle} \otimes_{S} b_{1\langle+\rangle} b_{2\langle-\rangle} \otimes_{S} b_{2\langle+\rangle} b_{3\langle-\rangle} \otimes_{S} \cdots \otimes_{S} b_{n-1\langle+\rangle} b_{n\langle-\rangle} \otimes_{S} b_{n\langle+\rangle} .
\end{aligned}
$$

Theorem 3.5. Let $\mathcal{K} T(S)^{\mathcal{B}}$ be a $\mathcal{K}$-equivariant $\mathcal{B}$-Galois extension, and $M$ be a right-left SAYD module over $\mathcal{K}$. Then $\omega_{*}$ defines an isomorphism of cyclic modules between ${ }_{\mathcal{K}} C_{*}(T, M)$ and $\widetilde{C}_{\mathcal{B}, *}(\mathcal{B}, \tilde{M})$, which are defined in (2.13) and (2.17) respectively. Here $\tilde{M}:=M \otimes \mathcal{K} T$ is the left-right $S A Y D$ module over $B$ introduced in Theorem 3.3.

Proof. The map $\omega_{n}$ is the composition of the following maps

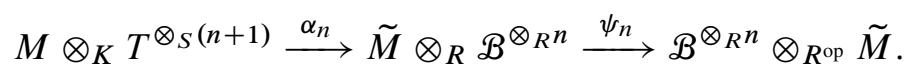

Here $\alpha_{*}$ and $\psi_{*}$ are given by

$\alpha_{n}\left(m \otimes \otimes_{\mathcal{K}} t_{0} \otimes_{S} \cdots \otimes_{S} t_{n}\right)$

$=m \otimes \mathcal{K} t_{0} t_{1\langle 0\rangle} \ldots t_{n\langle 0\rangle} \otimes_{R} t_{1\langle 1\rangle} t_{2\langle 1\rangle} \ldots t_{n\langle 1\rangle} \otimes_{R} \cdots \otimes_{R} t_{n-1\langle n-1\rangle} t_{n\langle n-1\rangle} \otimes_{R} t_{n\langle n\rangle}$,

$\alpha_{n}^{-1}\left(m \otimes \otimes_{\mathcal{K}} t \otimes_{R} b_{1} \otimes_{R} \cdots \otimes_{R} b_{n}\right)$

$=m \otimes_{\mathcal{K}} t b_{1\langle-\rangle} \otimes_{S} b_{1\langle+\rangle} b_{2\langle-\rangle} \otimes_{S} b_{2\langle+\rangle} b_{3\langle-\rangle} \otimes_{S} \cdots \otimes_{S} b_{n-1\langle+\rangle} b_{n\langle-\rangle} \otimes_{S} b_{n\langle+\rangle}$.

$\psi_{n}\left(m \otimes \otimes_{\mathcal{K}} t \otimes_{R} b_{1} \otimes_{R} \cdots \otimes_{R} b_{n}\right)=b_{1} \otimes_{R} \cdots \otimes_{R} b_{n} \otimes_{R^{\text {op }}} m \otimes_{\mathcal{K}} t$,

$\psi_{n}^{-1}\left(b_{1} \otimes_{R} \cdots \otimes_{R} b_{n} \otimes_{R}\right.$ op $\left.m \otimes_{\mathcal{K}} t\right)=m \otimes_{\mathcal{K}} t \otimes_{R} b_{1} \otimes_{R} \cdots \otimes_{R} b_{n}$.

One can easily use the $\mathcal{K}$-equivariant property of the coaction of $\mathscr{B}$ over $T$ and the $\mathcal{K}$-module algebra property of $T$ to see that the map $\alpha_{n}$ is well defined. Also using $\Delta(\mathfrak{s}(r))=1_{\mathcal{B}} \otimes_{R} \mathfrak{s}(r)$ and $r \triangleright\left(b_{1} \otimes_{R} \cdots \otimes_{R} b_{n}\right)=b_{1} \mathfrak{s}(r) \otimes_{R} \cdots \otimes_{R} b_{n}$, the morphism $\psi_{n}$ is obviously well defined. We show that $\omega$ is a map of cyclic modules. By multiplicity of the coproduct of $\mathcal{B}$, right $\mathcal{B}$-comodule algebra property of $T$ and the right $\times_{R}$-Hopf algebra property $b^{(2)} \mathrm{t}\left(\varepsilon\left(\mathrm{t}(r) b^{(1)}\right)\right)=\mathrm{t}(r) b$, the maps $\delta_{i}, 0 \leq i \leq n-1$ commute with $\omega$ as follows.

$$
\begin{aligned}
\delta_{i}\left(\omega_{n}\left(m \otimes_{\mathcal{K}} t_{0} \otimes_{S} \cdots \otimes_{S} t_{n}\right)\right) & \\
=\delta_{i} & \left(t_{1\langle 1\rangle} t_{2\langle 1\rangle} \ldots t_{n\langle 1\rangle} \otimes_{R} t_{2\langle 2\rangle} \ldots t_{n\langle 2\rangle} \otimes_{R} \ldots\right. \\
& \left.\ldots \otimes_{R} t_{n\langle n\rangle} \otimes_{R^{\mathrm{op}}}\left(m \otimes_{\mathcal{K}} t_{0} t_{1\langle 0\rangle} \ldots t_{n\langle 0\rangle}\right)\right) \\
= & t_{1\langle 1\rangle} t_{2\langle 1\rangle} \ldots t_{n\langle 1\rangle} \otimes_{R} \cdots \otimes_{R} \varepsilon\left(t_{i+1\langle i+1\rangle} \ldots t_{n\langle i+1\rangle}\right) \otimes_{R} \cdots \\
& \cdots \otimes_{R} t_{n\langle n\rangle} \otimes_{R^{\mathrm{op}}}\left(m \otimes_{\mathcal{K}} t_{0} t_{1\langle 0\rangle} \ldots t_{n\langle 0\rangle}\right) \\
= & t_{1\langle 1\rangle} t_{2\langle 1\rangle} \ldots t_{n\langle 1\rangle} \otimes_{R} \cdots \otimes_{R} t_{i\langle i\rangle} \ldots t_{n\langle i\rangle} \otimes_{R} t_{i+2\langle i+1\rangle} \ldots t_{n\langle i+1\rangle} \otimes_{R} \cdots \\
& \cdots \otimes_{R} t_{n\langle n-1\rangle} \otimes_{R^{\mathrm{op}}}\left(m \otimes_{\mathcal{K}} t_{0} t_{1\langle 0\rangle} \ldots t_{n\langle 0\rangle}\right)
\end{aligned}
$$




$$
\begin{aligned}
= & t_{1\langle 1\rangle} t_{2\langle 1\rangle} \ldots t_{n\langle 1\rangle} \otimes_{R} \cdots \otimes_{R}\left(t_{i} t_{i+1}\right)_{\langle i\rangle} t_{i+2\langle i\rangle} \ldots t_{n\langle i\rangle} \\
& \otimes_{R} t_{i+2\langle i+1\rangle} \ldots t_{n\langle i+1\rangle} \otimes_{R} \cdots \otimes_{R} t_{n\langle n-1\rangle} \\
& \otimes_{R^{\text {op }}}\left(m \otimes_{\mathcal{K}} t_{0} t_{1\langle 0\rangle} \cdots\left(t_{i} t_{i+1}\right)_{\langle 0\rangle} t_{i+2\langle 0\rangle} \ldots t_{n\langle 0\rangle}\right) \\
= & \omega_{n-1}\left(m \otimes \mathcal{K} t_{0} \otimes_{S} \cdots \otimes_{S} t_{i} t_{i+1} \otimes_{S} \cdots \otimes_{S} t_{n}\right) \\
= & \left.\omega_{n-1}\left(\delta_{i}\left(m \otimes \mathcal{K} t_{0} \otimes_{S} \cdots \otimes_{S} t_{n}\right)\right)\right) .
\end{aligned}
$$

Next we show that $\omega$ commutes with the last face morphism.

$$
\begin{aligned}
\delta_{n}\left(\omega_{n}\left(m \otimes_{\mathcal{K}} t_{0} \otimes_{S} \cdots \otimes_{S} t_{n}\right)\right) & \\
= & \delta_{n}\left(t_{1\langle 1\rangle} t_{2\langle 1\rangle} \ldots t_{n\langle 1\rangle} \otimes_{R} t_{2\langle 2\rangle} \ldots t_{n\langle 2\rangle} \otimes_{R} \ldots\right. \\
& \left.\ldots \otimes_{R} t_{n\langle n\rangle} \otimes_{R^{\mathrm{op}}}\left(m \otimes_{\mathcal{K}} t_{0} t_{1\langle 0\rangle} \ldots t_{n\langle 0\rangle}\right)\right) \\
= & \left(t_{1\langle 1\rangle} \ldots t_{n\langle 1\rangle} \otimes_{R} \cdots \otimes_{R} t_{n-1\langle n-1\rangle} t_{n\langle n-1\rangle}\right) \triangleleft t_{n\langle n\rangle}^{-} \\
& \otimes_{R^{\mathrm{op}}} t_{n\langle n\rangle}^{+} \triangleright\left(m \otimes_{\mathcal{K}} t_{0} t_{1\langle 0\rangle} \ldots t_{n\langle 0\rangle}\right) \\
= & \left(t_{1\langle 1\rangle} \ldots t_{n-1\langle 1\rangle} \otimes_{R} \cdots \otimes_{R} t_{n-1\langle n-1\rangle}\right) \triangleleft t_{n\langle 1\rangle} t_{n\langle 2\rangle}^{-} \\
& \otimes_{R^{\mathrm{op}}} t_{n\langle 2\rangle}^{+} \triangleright\left(m \otimes_{\mathcal{K}} t_{0} t_{1\langle 0\rangle} \ldots t_{n\langle 0\rangle}\right) \\
= & t_{1\langle 1\rangle} \ldots t_{n-1\langle 1\rangle} \otimes_{R} \cdots \otimes_{R} t_{n-1\langle n-1\rangle} \otimes_{R^{\mathrm{op}}} t_{n\langle 1\rangle} \triangleright\left(m \otimes_{\mathcal{K}} t_{0} t_{1\langle 0\rangle} \ldots t_{n\langle 0\rangle}\right) \\
= & t_{1\langle 1\rangle} \ldots t_{n-1\langle 1\rangle} \otimes_{R} \cdots \otimes_{R} t_{n-1\langle n-1\rangle} \otimes_{R^{\mathrm{op}}} \\
& m_{\langle 0\rangle} \otimes_{\mathcal{K}} t_{n\langle 1\rangle\langle+\rangle}\left(m_{\langle-1\rangle} \triangleright\left(t_{0} t_{1\langle 0\rangle} \ldots t_{n\langle 0\rangle} t_{n\langle 1\rangle\langle-\rangle}\right)\right) \\
= & t_{1\langle 1\rangle} \ldots t_{n-1\langle 1\rangle} \otimes_{R} \cdots \otimes_{R} t_{n-1\langle n-1\rangle} \otimes_{R^{\mathrm{op}}} \\
& m_{\langle 0\rangle} \otimes_{\mathcal{K}} t_{n}\left(m_{\langle-1\rangle} \triangleright\left(t_{0} t_{1\langle 0\rangle} \ldots t_{n-1\langle 0\rangle}\right)\right) \\
= & t_{1\langle 1\rangle} \ldots t_{n-1\langle 1\rangle} \otimes_{R} \cdots \otimes_{R} t_{n-1\langle n-1\rangle} \otimes_{R^{\mathrm{op}}} \\
& m_{\langle 0\rangle} \otimes_{\mathcal{K}} t_{n}\left(m_{\langle-n\rangle} \triangleright t_{0}\right)\left(m_{\langle-n+1\rangle} \triangleright t_{1\langle 0\rangle}\right) \ldots\left(m_{\langle-1\rangle} \triangleright t_{n-1\langle 0\rangle}\right) \\
= & \omega_{n-1}\left(m_{\langle 0\rangle} \otimes_{\mathcal{K}} t_{n}\left(m_{\langle-n\rangle} \triangleright t_{0}\right) \otimes_{S} m_{\langle-n+1\rangle} \triangleright t_{1} \otimes_{S} \cdots \otimes_{S} m_{\langle-1\rangle} \triangleright t_{n-1}\right) \\
= & \omega_{n-1} \delta_{n}\left(m \otimes \mathcal{K} t_{0} \otimes_{S} \cdots \otimes_{S} t_{n}\right) .
\end{aligned}
$$

We apply the right diagonal action of $\mathcal{B}$ over $\mathcal{B}^{\otimes_{R}(n-1)}$ in the third equality, the lemma [BS], 2.14 (ii), in the fourth equality, the action (3.2) in the fifth equality, the Lemma (3.2)(vi) in the sixth equality, the $\mathcal{K}$-module algebra property of $T$ in the seventh equality and $\mathcal{K}$-equivariant property of the coaction of $\mathcal{B}$ over $T$ in the eighth equality. By multiplicity of coproduct of $\mathscr{B}$ the commutativity of $\omega$ and $\sigma_{i}$, $0 \leq i \leq n-1$, can be verified as follows.

$$
\begin{aligned}
& \sigma_{i}\left(\omega_{n}\left(m \otimes_{\mathcal{K}} t_{0} \otimes_{S} \cdots \otimes_{S} t_{n}\right)\right) \\
& =\sigma_{i}\left(t_{1\langle 1\rangle} t_{2\langle 1\rangle} \ldots t_{n\langle 1\rangle} \otimes_{R} t_{2\langle 2\rangle} \ldots t_{n\langle 2\rangle} \otimes_{R} \ldots\right. \\
& \left.\quad \ldots \otimes_{R} t_{n\langle n\rangle} \otimes_{R^{\mathrm{op}}}\left(m \otimes_{\mathcal{K}} t_{0} t_{1\langle 0\rangle} \ldots t_{n\langle 0\rangle}\right)\right) \\
& =t_{1\langle 1\rangle} t_{2\langle 1\rangle} \ldots t_{n\langle 1\rangle} \otimes_{R}\left(t_{i\langle i+1\rangle} \ldots t_{n\langle i+1\rangle}\right)^{(1)} \otimes_{R}\left(t_{i+1\langle i+1\rangle} \ldots t_{n\langle i+1\rangle}\right)^{(2)} \otimes_{R} \ldots \\
& \quad \ldots \otimes_{R} t_{n\langle n\rangle} \otimes_{R^{\mathrm{op}}}\left(m \otimes_{\mathcal{K}} t_{0} t_{1\langle 0\rangle} \ldots t_{n\langle 0\rangle}\right)
\end{aligned}
$$




$$
\begin{aligned}
= & t_{1\langle 1\rangle} t_{2\langle 1\rangle} \ldots t_{n\langle 1\rangle} \otimes_{R} t_{i+1\langle i+1\rangle} \ldots t_{n\langle i+1\rangle} \otimes_{R} t_{i+1\langle i+2\rangle} \ldots t_{n\langle i+2\rangle} \otimes_{R} \cdots \\
& \quad \cdots \otimes_{R} t_{n\langle n+1\rangle} \otimes_{R^{\text {op }}}\left(m \otimes_{\mathcal{K}} t_{0} t_{1\langle 0\rangle} \ldots t_{n\langle 0\rangle}\right) \\
= & \omega_{n+1}\left(m \otimes_{\mathcal{K}} t_{0} \otimes_{S} \ldots t_{i} \otimes_{S} 1_{T} \otimes_{S} t_{i+1} \otimes_{S} \cdots \otimes_{S} t_{n} \otimes_{S}\right) \\
= & \omega_{n+1}\left(\sigma_{i}\left(m \otimes \mathcal{K} t_{0} \otimes_{S} \cdots \otimes_{S} t_{n}\right)\right) .
\end{aligned}
$$

Using Lemma 2.14 (iv) in [BS], the commutativity of $\omega$ with the last degeneracy follows from

$$
\begin{aligned}
& \sigma_{n}\left(\omega_{n}\left(m \otimes_{\mathcal{K}} t_{0} \otimes_{S} \cdots \otimes_{S} t_{n}\right)\right) \\
& =\sigma_{n}\left(t_{1\langle 1\rangle} t_{2\langle 1\rangle} \ldots t_{n\langle 1\rangle} \otimes_{R} t_{2\langle 2\rangle} \ldots t_{n\langle 2\rangle} \otimes_{R} \ldots\right. \\
& \left.\quad \ldots \otimes_{R} t_{n\langle n\rangle} \otimes_{R^{\text {op }}}\left(m \otimes_{\mathcal{K}} t_{0} t_{1\langle 0\rangle} \ldots t_{n\langle 0\rangle}\right)\right) \\
& =t_{1\langle 1\rangle} t_{2\langle 1\rangle} \ldots t_{n\langle 1\rangle} \otimes_{R} t_{2\langle 2\rangle} \ldots t_{n\langle 2\rangle} \otimes_{R} \cdots \\
& \quad \cdots \otimes_{R} t_{n\langle n\rangle} \otimes_{R} 1_{T} \otimes_{R^{\text {op }}}\left(m \otimes_{\mathcal{K}} t_{0} t_{1\langle 0\rangle} \ldots t_{n\langle 0\rangle}\right) \\
& =\omega_{n+1}\left(m \otimes_{\mathcal{K}} t_{0} \otimes_{S} \cdots \otimes_{S} t_{n} \otimes_{S} 1_{T}\right) \\
& =\omega_{n+1}\left(\sigma_{n}\left(m \otimes_{\mathcal{K}} t_{0} \otimes_{S} \cdots \otimes_{S} t_{n}\right)\right) .
\end{aligned}
$$

Finally, we show that $\omega$ commutes with the cyclic maps.

$$
\begin{aligned}
& \tau_{n}\left(\omega_{n}\left(m \otimes \mathcal{K} t_{0} \otimes_{S} \cdots \otimes_{S} t_{n}\right)\right) \\
& =\tau_{n}\left(t_{1\langle 1\rangle} t_{2\langle 1\rangle} \ldots t_{n\langle 1\rangle} \otimes_{R} t_{2\langle 2\rangle} \ldots t_{n\langle 2\rangle} \otimes_{R} \ldots\right. \\
& \left.\cdots \otimes_{R} t_{n\langle n\rangle} \otimes_{R^{\mathrm{op}}}\left(m \otimes_{\mathcal{K}} t_{0} t_{1\langle 0\rangle} \ldots t_{n\langle 0\rangle}\right)\right) \\
& =\left(t_{0\langle 1\rangle} t_{1\langle 0\rangle\langle 1\rangle} \ldots t_{n\langle 0\rangle\langle 1\rangle} \otimes_{R} t_{1\langle 1\rangle} \ldots t_{n\langle 1\rangle} \otimes_{R} \ldots\right. \\
& \left.\cdots \otimes_{R} t_{n-1\langle n-1\rangle} t_{n\langle n-1\rangle}\right) \triangleleft t_{n\langle n\rangle}^{-} \otimes_{R^{\mathrm{op}}} t_{n\langle n\rangle}^{+} \triangleright\left(m \otimes \mathcal{K} t_{0\langle 0\rangle} t_{1\langle 0\rangle\langle 0\rangle} \ldots t_{n\langle 0\rangle\langle 0\rangle}\right) \\
& =\left(t_{0\langle 1\rangle} t_{1\langle 1\rangle} \ldots t_{n\langle 1\rangle} \otimes_{R} t_{1\langle 2\rangle} \ldots t_{n\langle 2\rangle} \otimes_{R} \ldots\right. \\
& \left.\cdots \otimes_{R} t_{n-1\langle n\rangle} t_{n\langle n\rangle}\right) \triangleleft t_{n\langle n+1\rangle}^{-} \otimes_{R^{\text {op }}} t_{n\langle n+1\rangle}^{+} \triangleright\left(m \otimes \mathcal{K} t_{0\langle 0\rangle} t_{1\langle 0\rangle} \ldots t_{n\langle 0\rangle}\right) \\
& =\left(t_{0\langle 1\rangle} t_{1\langle 1\rangle} \ldots t_{n-1\langle 1\rangle} \otimes_{R} t_{1\langle 2\rangle} \ldots t_{n-1\langle 2\rangle} \otimes_{R} \ldots\right. \\
& \left.\cdots \otimes_{R} t_{n-1\langle n\rangle}\right) \triangleleft t_{n\langle 1\rangle} t_{n\langle 2\rangle}^{-} \otimes_{R^{\mathrm{op}}} t_{n\langle 2\rangle}^{+} \triangleright\left(m \otimes \mathcal{K} t_{0\langle 0\rangle} t_{1\langle 0\rangle} \ldots t_{n\langle 0\rangle}\right) \\
& =t_{0\langle 1\rangle} t_{1\langle 1\rangle} \ldots t_{n-1\langle 1\rangle} \otimes_{R} t_{1\langle 2\rangle} \ldots t_{n-1\langle 2\rangle} \otimes_{R} \cdots \otimes_{R} t_{n-1\langle n\rangle} \\
& \otimes_{R^{\text {op }}} t_{n\langle 1\rangle} \triangleright\left(m \otimes \mathcal{K} t_{0\langle 0\rangle} t_{1\langle 0\rangle} \ldots t_{n\langle 0\rangle}\right) \\
& =t_{0\langle 1\rangle} t_{1\langle 1\rangle} \ldots t_{n-1\langle 1\rangle} \otimes_{R} t_{1\langle 2\rangle} \ldots t_{n-1\langle 2\rangle} \otimes_{R} \cdots \otimes_{R} t_{n-1\langle n\rangle} \\
& \otimes_{R^{\mathrm{op}}} m_{\langle 0\rangle} \otimes_{\mathcal{K}} t_{n\langle 1\rangle\langle+\rangle}\left(m _ { \langle - 1 \rangle } \triangleright \left(t_{0\langle 0\rangle} t_{1\langle 0\rangle} \ldots t_{n\langle 0\rangle} t_{n\langle 1\rangle\langle-\rangle}\right.\right. \\
& =t_{0\langle 1\rangle} t_{1\langle 1\rangle} \ldots t_{n-1\langle 1\rangle} \otimes_{R} t_{1\langle 2\rangle} \ldots t_{n-1\langle 2\rangle} \otimes_{R} \cdots \otimes_{R} t_{n-1\langle n\rangle} \\
& \otimes_{R^{\mathrm{op}}} m_{\langle 0\rangle} \otimes_{\mathcal{K}} t_{n}\left(m_{\langle-n\rangle} \triangleright t_{0\langle 0\rangle}\right)\left(m_{\langle-n+1\rangle} t_{1\langle 0\rangle}\right) \ldots\left(m_{\langle-1\rangle} t_{n-1\langle 0\rangle}\right) \\
& =\omega_{n}\left(m_{\langle 0\rangle} \otimes \mathcal{K} t_{n} \otimes_{S} m_{\langle-n\rangle} t_{0} \otimes_{S} \cdots \otimes_{S} m_{\langle-1\rangle} t_{n-1}\right) \\
& =\omega_{n} \tau_{n}\left(m \otimes \mathcal{K} t_{0} \otimes S \cdots \otimes{ }_{S} t_{n}\right) \text {. }
\end{aligned}
$$

We use the coaction property of $T$ over $\mathscr{B}$ in the second equality, the right diagonal action of $\mathcal{B}$ over $\mathcal{B}^{\otimes_{R}}$ in the third equality, Lemma 2.14 (ii) of [BS] in the fourth 
equality, the action (3.2) in the fifth equality, Lemma 3.2 (vi) in the sixth equality, the $\mathcal{K}$-module algebra property of $T$ in the seventh equality and $\mathcal{K}$-equivariant property of the coaction of $\mathscr{B}$ over $T$ in the eighth equality.

3.2. Examples. As first example, one notes that by using Proposition 2.6 and Theorem 3.3, for $\mathcal{K}=S \otimes S^{\text {op }}$ and $M=S$, we see that Theorem 3.5 generalizes Theorem 2.6 in [BS].

We like to illustrate the SAYD module constructed in Theorem 3.3 by an explicit and nontrivial example. Let $\mathscr{H}$ and $\mathscr{F}$ denote two Hopf algebras such that $\mathscr{H}$ acts on $\mathscr{F}$ and makes it a left module algebra. We let $A:=\mathscr{F} \rtimes \mathscr{H}$ be the usual crossed product algebra, that is, $\mathscr{F} \otimes \mathscr{H}$ as a vector space with multiplication $(f \rtimes h)(g \rtimes v)=$ $f\left(h_{(1)} \triangleright g\right) \rtimes h_{(2)} v$, and $1 \rtimes 1$ as its unit. One knows that $\mathbf{\nabla}: A \rightarrow A \otimes \mathscr{H}$ defined by $\mathbf{\nabla}(f \rtimes h)=f \rtimes h_{(1)} \otimes h_{(2)}$ defines a comodule algebra over the Hopf algebra $\mathscr{H}$. One also easily verifies that $B:=A^{\mathscr{H}}=\mathscr{F} \otimes \mathbb{C} \simeq \mathscr{F}$, and $A(B)^{\mathscr{H}}$ is a Hopf Galois extension. We let $\mathcal{K}:=B \otimes \mathscr{F} \otimes B^{\circ}$ be the usual left $\times_{B}$-Hopf algebra, i.e., as an algebra it is tensor product of algebras and its coring structure is given by

$$
\begin{aligned}
\mathfrak{s}(b) & =b \otimes 1 \otimes 1, \\
\mathrm{t}(b) & =1 \otimes 1 \otimes b, \\
\Delta\left(b_{1} \otimes f \otimes b_{2}\right) & =b_{1} \otimes f_{(1)} \otimes 1 \otimes_{B} 1 \otimes f_{(2)} \otimes b_{2}, \\
\varepsilon\left(b_{1} \otimes f \otimes b_{2}\right) & =\varepsilon(f) b_{1} b_{2},
\end{aligned}
$$

One observes that

$$
v^{-1}\left(b_{1} \otimes f \otimes b_{2} \otimes_{B^{o}} b_{3} \otimes g \otimes b_{4}\right)=b_{1} \otimes f_{(1)} \otimes 1 \otimes_{B} b_{2} b_{3} \otimes S\left(f_{(2)}\right) g \otimes b_{4} .
$$

So we see that

$$
\left(b_{1} \otimes f \otimes b_{2}\right)^{-} \otimes_{B}\left(b_{1} \otimes f \otimes b_{2}\right)^{+}=b_{1} \otimes f_{(1)} \otimes 1 \otimes_{B} b_{2} \otimes S\left(f_{(2)}\right) \otimes 1 .
$$

Now let $N$ be a right-left SAYD module over the Hopf algebra $\mathcal{F}$ and let also $x \otimes x^{-1} \in B \otimes B^{o}$ be a group-like element with $x \in Z(B)$, the center of $B$. We define the following action and coaction on $M:={ }^{x \otimes x^{-1}} B \otimes N=B \otimes N$.

$$
\begin{aligned}
\mathbf{\nabla}(b \otimes n) & =b x \otimes n_{\langle-1\rangle} \otimes x^{-1} \otimes_{B} 1 \otimes n_{\langle 0\rangle}, \\
(b \otimes n) \cdot\left(b_{1} \otimes f \otimes b_{2}\right) & =b_{2} b b_{1} \otimes n \cdot f
\end{aligned}
$$

Lemma 3.6. Via the above action and coaction ${ }^{x \otimes x^{-1}} B \otimes N$ is a right-left $S A Y D$ module over $\mathcal{K}$.

Proof. It is obvious that the action and coaction are well defined. In the following 
we check the AYD condition:

$$
\begin{aligned}
\boldsymbol{\nabla}( & \left.(b \otimes n) \cdot\left(b_{1} \otimes f \otimes b_{2}\right)\right) \\
= & \nabla\left(b_{2} b b_{1} \otimes n \cdot f\right) \\
= & b_{2} b b_{1} x \otimes(n f)_{\langle-1\rangle} \otimes x^{-1} \otimes_{B} 1 \otimes(n \cdot f)_{\langle 0\rangle} \\
= & b_{2} b b_{1} x \otimes S\left(f_{(3)}\right) n_{\langle-1\rangle} f_{(1)} \otimes x^{-1} \otimes_{B} 1 \otimes n_{\langle 0\rangle} \cdot f_{(2)} \\
= & \left(b_{2} \otimes S\left(f_{(3)}\right) \otimes 1\right)\left(b x \otimes n_{\langle-1\rangle} \otimes x^{-1}\right)\left(b_{1} \otimes f_{(1)} \otimes 1\right) \otimes_{B}\left(1 \otimes n_{\langle 0\rangle}\right) \\
& \cdot\left(1 \otimes f_{(2)} \otimes 1\right) \\
= & k_{(2)}^{+} n_{\langle-1\rangle} k_{(1)} \otimes_{B} n_{\langle 0\rangle} \cdot k_{(2)}^{-} .
\end{aligned}
$$

The stability condition follows from the stability of $N$.

One then defines a left action of $\mathcal{K}$ on $A$ by

$$
\left(b_{1} \otimes f \otimes b_{2}\right) \triangleright(g \rtimes h)=b_{1} f_{(1)} g\left(h_{(1)} \triangleright\left(S\left(f_{(2)}\right) b_{2}\right)\right) \rtimes h_{(2)} .
$$

Lemma 3.7. Let $\mathcal{F}$ be commutative. Then the above action makes $A$ a $\mathcal{K}$-module algebra.

Proof. Let us first prove that the equation (3.4) defines an action. Indeed for $k_{1}:=$ $b_{1} \otimes e \otimes b_{2}$ and $k_{2}:=b_{3} \otimes f \otimes b_{4}$, by using the facts that $\mathcal{F}$ is commutative and an $\mathscr{H}$-module algebra we have

$$
\begin{aligned}
k_{1} \triangleright\left(k_{2} \triangleright(g \rtimes h)\right) & =k_{1} \triangleright\left(b_{3} f_{(1)} g\left(h_{(1)} \triangleright\left(S\left(f_{(2)}\right) b_{4}\right)\right) \rtimes h_{(2)}\right) \\
& =\left(b _ { 1 } b _ { 3 } e _ { ( 1 ) } f _ { ( 1 ) } g \left(h_{(1)} \triangleright\left(S\left(f_{(2)} b_{4}\right) h_{(2)} \triangleright\left(S\left(e_{2}\right) b_{2}\right) \rtimes h_{(2)}\right)\right.\right. \\
& =k_{1} k_{2} \triangleright(g \rtimes h) .
\end{aligned}
$$

Obviously the action defined in (3.4) is unital. We use $h \triangleright 1_{\mathscr{F}}=\varepsilon(h) 1_{\mathscr{F}}$ to see that

$$
\begin{aligned}
& \mathfrak{s}(b) \triangleright(f \rtimes h)=(b \otimes 1 \otimes 1) \triangleright(f \rtimes h)=b f \rtimes h . \\
& \mathfrak{t}(b) \triangleright(f \rtimes h)=(1 \otimes 1 \otimes b) \triangleright(g \rtimes v)=g\left(h_{(1)} \triangleright b\right) \rtimes h_{(2)} .
\end{aligned}
$$

This tells us that the multiplication of $\mathcal{K}$ is $B$-balanced. Now we show that $k \triangleright$ $\left(a_{1} a_{2}\right)=\left(k_{(1)} \triangleright a_{1}\right)\left(k_{(2)} \triangleright a_{2}\right)$. Let $k=\left(b_{1} \otimes f \otimes b_{2}\right), a_{1}=g \rtimes h$ and $a_{2}=l \rtimes v$. Then we have

$$
\begin{aligned}
k \triangleright\left(a_{1} a_{2}\right) & =k \triangleright\left(g h_{(1)} \triangleright l \rtimes h_{(2)} v\right) \\
& =b_{1} f_{(1)} g\left(h_{(1)} \triangleright l\right)\left(h_{(2)} v_{(1)} \triangleright\left(S\left(f_{(2)}\right) b_{2}\right)\right) \rtimes h_{(3)} v_{(2)}
\end{aligned}
$$


On the other hand by using the fact that $\mathcal{F}$ is an $\mathscr{H}$-module algebra we see

$$
\begin{aligned}
& \left(k_{(1)} \triangleright a_{1}\right)\left(k_{(2)} \triangleright a_{2}\right) \\
& =\left(\left(b_{1} \otimes f_{(1)} \otimes 1\right) \triangleright(g \rtimes h)\right)\left(\left(1 \otimes f_{(2)} \otimes b_{2}\right) \triangleright(l \rtimes v)\right) \\
& =\left(b_{1} f_{(1)} g\left(h_{(1)} \triangleright S\left(f_{(2)}\right)\right) \rtimes h_{(2)}\right)\left(f_{(3)} l\left(v_{(1)} \triangleright\left(S\left(f_{(4)}\right) b_{2}\right)\right) \rtimes v_{(2)}\right) \\
& =b_{1} f_{(1)} g\left(h_{(1)} \triangleright S\left(f_{(2)}\right)\right)\left(h_{(2)} \triangleright\left(f_{(3)} l\left(v_{(1)} \triangleright\left(S\left(f_{(4)}\right) b_{2}\right)\right)\right) \rtimes h_{(3)} v_{(2)}\right. \\
& =b_{1} f_{(1)} g\left(h_{(1)} \triangleright S\left(f_{(2)}\right)\right)\left(h_{(2)} \triangleright f_{(3)}\right)\left(h_{(3)} \triangleright l\right)\left(h_{(4)} v_{(1)} \triangleright\left(S\left(f_{(4)}\right) b_{2}\right)\right) \rtimes h_{(5)} v_{(2)} \\
& =b_{1} f_{(1)} g\left(h_{(1)} \triangleright l\right)\left(h_{(2)} v_{(1)} \triangleright\left(S\left(f_{(2)}\right) b_{2}\right)\right) \rtimes h_{(3)} v_{(2)} .
\end{aligned}
$$

Finally we check the condition (2.11). For $k:=b_{1} \otimes f \otimes b_{2}$ we have

$$
\begin{aligned}
k \triangleright 1_{A} & =\left(b_{1} \otimes f \otimes b_{2}\right) \triangleright(1 \rtimes 1) \\
& =b_{1} f_{(1)}\left(S\left(f_{(2)}\right) b_{2}\right) \rtimes 1 \\
& =\varepsilon(f) b_{1} b_{2} \rtimes 1 \\
& =\left(\varepsilon(f) b_{1} b_{2} \otimes 1 \otimes 1\right) \triangleright(1 \rtimes 1) \\
& =\Im(\varepsilon(k)) \triangleright 1_{A} .
\end{aligned}
$$

One easily sees that the coaction $\mathbf{\nabla}: A \rightarrow A \otimes \mathscr{H}$ is $\mathcal{K}$ equivariant.

We now let the group of all unit elements of $B$, which is denoted by $B^{\times}$. Define a category whose objects and morphisms are both elements of $B^{\times}$. More precisely, for $b_{1}, b_{2} \in B^{\times}, \operatorname{Mor}\left(b_{1}, b_{2}\right)$ consists the unique element denoted by $\hat{c}$, where $c=b_{2} b_{1}^{-1}$. We let $B^{\times} \times{ }^{\mathscr{F}} \mathrm{SAY}_{\mathscr{F}}$ be the product of categories. Here ${ }^{\mathscr{F}} \mathrm{SAYD}_{\mathscr{F}}$ is the category whose objects are left-right SAYD modules over $\mathscr{F}$ and morphisms are $\mathscr{F}$-linear and $\mathscr{F}$-colinear maps. We define the following functor:

$$
\begin{aligned}
& \Phi: B^{\times} \times{ }^{\mathscr{F}} \mathrm{SAYD}_{\mathscr{F}} \rightarrow \mathcal{K} \operatorname{SAYD}(\mathcal{H})^{\mathcal{K}}, \\
& \Phi((x, N))={ }^{x \otimes x^{-1}} B \otimes N, \\
& \Phi(\hat{y}, \phi):{ }^{x \otimes x^{-1}} B \otimes N_{1} \rightarrow{ }^{x y \otimes(x y)^{-1}} B \otimes N_{2}, \\
& \Phi(\hat{y}, \phi)(b \otimes n)=b y^{-1} \otimes \phi(n) .
\end{aligned}
$$

Here $y \in B^{\times}$and $\phi \in{ }^{\mathscr{F}} \operatorname{Hom}_{\mathscr{F}}\left(N_{1}, N_{2}\right)$.

Proposition 3.8. The above assignment $\Phi$ defines a covariant functor.

Proof. We need to show that $\Phi$ is a morphism map. Indeed, first we see that $\Phi(\hat{y}, \phi)$ is a $\mathcal{K}$-colinear map. Using the facts that $\phi$ is an $\mathcal{F}$-colinear map, that $B$ is commutative, 
and also the bialgebroid structure of $\mathcal{K}=B \otimes \mathcal{F} \otimes B$, we see

$$
\begin{aligned}
\boldsymbol{\nabla}(\Phi(\hat{y}, \phi)(b \otimes n)) & =\boldsymbol{\nabla}\left(b y^{-1}, \phi(n)\right) \\
& =b y^{-1} x y \otimes \phi(n)_{\langle-1\rangle} \otimes(x y)^{-1} \otimes_{B} 1 \otimes \phi(n)_{\langle 0\rangle} \\
& =b x \otimes \phi\left(n_{\langle-1\rangle}\right) \otimes x^{-1} y^{-1} \otimes_{B} 1 \otimes \phi\left(n_{\langle 0\rangle}\right) \\
& =b x \otimes \phi\left(n_{\langle-1\rangle}\right) \otimes x^{-1} \otimes_{B} y^{-1} \otimes \phi\left(n_{\langle 0\rangle}\right) \\
& =\left(\operatorname{Id}_{\mathcal{K}} \otimes \Phi(\hat{y}, \phi)\right)(\nabla(b \otimes n)) .
\end{aligned}
$$

Now we prove that $\Phi(\hat{y}, \phi)$ is a $\mathcal{K}$-linear map.

$$
\begin{aligned}
\Phi(\hat{y}, \phi)\left((b \otimes n) \cdot\left(b_{1} \otimes f \otimes b_{2}\right)\right) & =\Phi(\hat{y}, \phi)\left(b_{2} b b_{1} \otimes n \cdot f\right) \\
& =\left(b_{2} b b_{1} y^{-1} \otimes \phi(n \cdot f)\right) \\
& =\left(b_{2} b b_{1} y^{-1} \otimes \phi(n) \cdot f\right) \\
& =\left(b_{2} b b_{1} y^{-1} \otimes \phi(n \cdot f)\right) \\
& =(\Phi(\hat{y}, \phi))\left(b_{2} b b_{1} \otimes n \cdot f\right) .
\end{aligned}
$$

Finally one uses again the commutativity of $B$ to see that $\Phi\left(\left(\widehat{y_{1}}, \phi_{1}\right) \circ\left(\widehat{y_{2}}, \phi_{2}\right)\right)=$ $\Phi\left(\widehat{y_{1} y_{2}}, \phi_{1} \phi_{2}\right)=\Phi\left(\widehat{y_{1}}, \phi_{1}\right) \circ \Phi\left(\widehat{y_{2}}, \phi_{2}\right)$.

As a result one composes the functors $\Phi$ and $F$ defined in Propositions 3.4 and 3.8 respectively to get the following functor

$$
F \circ \Phi: B^{\times} \times{ }^{\mathscr{F}} \mathrm{SAYD}_{\mathscr{F}} \rightarrow \mathscr{H} \operatorname{SAYD}^{\mathscr{H}}
$$

One notes that in the simplest possible case of this example, i.e, when $\mathscr{F}:=B=$ $\mathbb{C}, N:=\mathbb{C}, x=1_{\mathbb{C}}$ the resulting SAYD module is $\mathscr{H}$ with the standard action and coaction, i.e, the action and coaction are defined by the adjoint action and the comultiplication of $\mathscr{H}$ respectively.

\section{References}

[BS] G. Böhm and D. Ştefan, (Co)cyclic (co)homology of bialgebroids: an approach via (co)monads. Comm. Math. Phys. 282 (2008), 239-286. Zbl 1153.18004 MR 2415479

[B] G. Böhm, Hopf algebroids. In Handbook of Algebra, Vol. 6, Elsevier/North-Holland, Amsterdam 2009, 173-235. Zbl 1220.16022 MR 2553659

[BW] T. Brzezinski and R. Wisbauer, Corings and comodules. London Math. Soc. Lecture Note Ser. 309, Cambridge University Press, Cambridge 2003. Zbl 1035.16030 MR 2012570

[C-Book] A. Connes, Noncommutative geometry. Academic Press, San Diego, CA, 1994. Zbl 0818.46076 MR 1303779 
[CM98] A. Connes and H. Moscovici, Hopf algebras, cyclic cohomology and the transverse index theorem. Comm. Math. Phys. 198 (1998), 199-246. Zbl 0940.58005 MR 1657389

[CM00] A. Connes and H. Moscovici, Cyclic cohomology and Hopf algebra symmetry. Lett. Math. Phys. 52 (2000), 1-28. Zbl 0974.58006 MR 1800488

[CM01] A. Connes and H. Moscovici, Differentiable cyclic cohomology and Hopf algebraic structures in transverse geometry. In Essays on geometry and related topics, Vol. 1, Monogr. Enseign. Math. 38, Enseignement Math., Geneva 2001, 217-255. Zbl 1018.57013 MR 1929328

[Cra] M. Crainic, Cyclic cohomology of étale groupoids: the general case. K-Theory 17 (1999), 319-362. Zbl 0937.19007 MR 1706117

[Gor] A. Gorokhovsky, Secondary characteristic classes and cyclic cohomology of Hopf algebras. Topology 41 (2002), 993-1016. Zbl 1008.58008 MR 1923996

[HKRS1] P. M. Hajac, M. Khalkhali, B. Rangipour, and Y. Sommerhäuser, Stable antiYetter-Drinfeld modules. C. R. Math. Acad. Sci. Paris 338 (2004), 587-590. Zbl 1060.16037 MR 2056464

[HKRS2] P. M. Hajac, M. Khalkhali, B. Rangipour, and Y. Sommerhäuser, Hopf-cyclic homology and cohomology with coefficients. C. R. Math. Acad. Sci. Paris 338 (2004), 667-672. Zbl 1064.16006 MR 2065371

[JS] P. Jara and D. Ştefan, Hopf-cyclic homology and relative cyclic homology of Hopf-Galois extensions. Proc. London Math. Soc. (3) 93 (2006), 138-174. Zbl 1158.16007 MR 2235945

[Kay05] A. Kaygun, Bialgebra cyclic homology with coefficients. K-Theory 34 (2005), 151-194. Zbl 1114.16011 MR 2180111

[Kay06] A. Kaygun, The universal Hopf-cyclic theory. J. Noncommut. Geom. 2 (2008), 333-351. Zbl 1189.16011 MR 2411421

[KP] M. Khalkhali and A. Pourkia, Hopf cyclic cohomology in braided monoidal categories. Homology, Homotopy Appl. 12 (2010), 111-155. Zbl 1209.58008 MR 2607413

[KR02] M. Khalkhali and B. Rangipour, A new cyclic module for Hopf algebras. $K$-Theory 27 (2002), 111-131. Zbl 1020.16029 MR 1942182

[KR03] M. Khalkhali and B. Rangipour, Invariant cyclic homology. K-Theory 28 (2003), 183-205. Zbl 1028.58006 MR 1995876

[KR04] M. Khalkhali and B. Rangipour, Para-Hopf algebroids and their cyclic cohomology. Lett. Math. Phys. 70 (2004), 259-272. Zbl 1067.58007 MR 2128954

[KR05] M. Khalkhali and B. Rangipour, A note on cyclic duality and Hopf algebras. Comm. Algebra 33 (2005), 763-773. Zbl 1089.16011 MR 2128410

[Lo] J.-L. Loday, Cyclic homology. 2nd ed., Grundlehren Math. Wiss. 301, SpringerVerlag, Berlin 1998. Zbl 0885.18007 MR 1600246

[Ra] B. Rangipour, Cyclic cohomology of corings. J. K-Theory 4 (2009), 193-207. Zbl 1192.16008 MR 2538722 
[Sch98] P. Schauenburg, Bialgebras over noncommutative rings and a structure theorem for Hopf bimodules. Appl. Categ. Structures 6 (1998), 193-222. Zbl 0908.16033 MR 1629385

[Sch] P. Schauenburg, Duals and doubles of quantum groupoids $\left(\times_{R}\right.$-Hopf algebras). In New trends in Hopf algebra theory (La Falda, 1999), Contemp. Math. 267, Amer. Math. Soc., Providence, RI, 2000, 273-299. Zbl 0974.16036 MR 1800718

Received November 23, 2010

M. Hassanzadeh, Institut des Hautes Études Scientifiques, 35 Route de Chartres, 91440 Bures Sur Yvette, France

E-mail: mhassanz@ihes.fr

B. Rangipour, Department of Mathematics and Statistics, University of New Brunswick, Fredericton, NB, Canada E3B 5A3

E-mail: bahram@unb.ca 